\title{
Structure and mechanism of a primate ferroportin
}

3 Zhenning Ren ${ }^{1, *}$, Shuai Gao ${ }^{2, *}$, Jiemin Shen ${ }^{1, *}$, Lie Wang ${ }^{1}$, Zhichun $\mathrm{Xu}^{1}$, Ye Yu ${ }^{1}$, Preetham

4 Bachina $^{1}$, Hanzhi Zhang ${ }^{1}$, Arthur Laganowsky ${ }^{3}$, Nieng Yan $^{2}$, Ming Zhou ${ }^{1, \#, ~ Y a p i n g ~ P a n ~}{ }^{1, * \#}$

$5 \quad{ }^{1}$ Verna and Marrs McLean Department of Biochemistry and Molecular Biology, Baylor College

6 of Medicine, Houston, TX 77030, USA

$7 \quad{ }^{2}$ Department of Molecular Biology, Princeton University, Princeton, NJ 08544, USA

$8{ }^{3}$ Department of Chemistry, Texas A \& M University, College Station, TX 77843, USA

9

$10 *$ These authors contributed equally to the work.

11 \# Correspondence to M. Zhou (mzhou@bcm.edu) and Y. Pan (yaping.pan @ bcm.edu) 


\section{Abstract}

15 Ferroportin is the only cellular iron exporter in human and essential for iron homoeostasis.

16 Mutations in ferroportin are associated with hemochromatosis or ferroportin diseases

17 characterized by a paradoxical combination of anemia and abnormal accumulation of iron in

18 cells. Ferroportin is also the target of hepcidin, which is a hormone that downregulates

19 ferroportin activity. However, due to a lack of three-dimensional structures, the mechanism of

20 iron transport by ferroportin and its regulation by hepcidin remains unclear. Here we present the

21 structure of a ferroportin from the primate Philippine tarsier (TsFpn) at $3.0 \AA$ A resolution

22 determined by cryo-electron microscopy. TsFpn has a structural fold common to major facilitator

23 superfamily of transporters and the current structure is in an outward-open conformation. The

24 structure identifies two potential ion binding sites with each site coordinated by two residues.

25 Functional studies demonstrate that TsFpn is a $\mathrm{H}^{+} / \mathrm{Fe}^{2+}$ antiporter and that transport of one $\mathrm{Fe}^{2+}$ is

26 coupled to the transport of two $\mathrm{H}^{+}$in the opposite direction such that the transport cycle is

27 electroneutral. Further studies show that the two ion binding sites affect transport of $\mathrm{H}^{+}$and $\mathrm{Fe}^{2+}$

28 differently. The structure also provides mechanistic interpretation for mutations that cause

29 ferroportin diseases. 


\section{Introduction}

In mammals, ferroportin (Fpn) exports cellular iron and is highly expressed in enterocytes,

34 hepatocytes and macrophages to distribute iron absorbed from food or recovered from digestion of senescent red blood cells ${ }^{1}$. Fpn also mediates iron transport across the placenta and thus is required for the normal development of embryos ${ }^{2}$. Fpn activity is regulated by hepcidin, a

37 peptide hormone, which reduces Fpn activity by a combination of inhibiting the transport activity

38 and promoting endocytosis of $\mathrm{Fpn}^{3,4}$. More than sixty Fpn mutations have been identified in

39 human to cause ferroportin diseases ${ }^{5,6}$ that are characterized by accumulation of iron inside of macrophages, highlighting its important physiological role in iron homeostasis. Fpn and its modulation by hepcidin has been the focus of targeted therapeutics for treating ferroportin 42 diseases $^{7-9}$.

44 Fpn belongs to the solute carrier family 40 (SLC40A1) ${ }^{10-12}$ and is a member of the major

46 (GLUT1-5) $^{13-15}$, peptide transporter (PEPT1, SLC15A1) ${ }^{16-18}$, and equilibrative nucleoside

47 transporter ${ }^{19}$. Transporters of the MFS family share a common structural fold that has two

48 homologous halves forming a clam-shell like architecture. A single substrate binding site is

49 commonly located to the center of the clam-shell, and substrate translocation is achieved by

50 rock-switch type motions of the two halves of the clam-shell so that the substrate binding site is

51 alternatively exposed to either side of the membrane $e^{20}$. Structures of a bacterial homolog of Fpn

52 (Bdellovibrio bacteriovorous; BbFpn) were reported recently ${ }^{21,22}$, which enhances our

53 understanding of Fpn. However, BbFpn has 20\% sequence identity and $49 \%$ similarity to that 
54 of human Fpn and may not depict an accurate representation of the mechanism of iron

55 recognition and transport in human Fpn. We expressed and purified an Fpn from Philippine

56 tarsier (Tarcius syrichta or Carlito syrichta; TsFpn), which is $92 \%$ identical and $98 \%$ similar to

57 human Fpn, and we characterized its function and determined its structure.

In vitro functional studies of TsFpn

TsFpn is expressed and purified from insect cells and elutes as a single peak on a size-exclusion

62 chromatography column. The elution volume is consistent with TsFpn being a monomer (Fig. 1a and Methods). TsFpn has three predicted N-linked glycosylation sites $^{23}$ and migrates as a diffused band on an SDS-PAGE, and addition of glycosidases PNGase F and EndoH helps focus the protein band (Fig. 1a), confirming that TsFpn is glycosylated.

67 We reconstituted the purified TsFpn protein into liposomes and measured transport of $\mathrm{Fe}^{2+}$ using a flux assay (Fig. 1b and Methods). Liposomes reconstituted with TsFpn show significant Fe ${ }^{2+}$

69 uptake while liposomes without the protein do not have significant change of fluorescence over

70 time (Fig. 1c). Since $\mathrm{Fe}^{2+}$ is easily oxidized under aerobic conditions, a reducing reagent $(1 \mathrm{mM}$

71 vitamin C) was added to the external solution to stabilize ferrous. Although ferrous transport can

72 be observed, the addition of reducing reagents affects free $\mathrm{Fe}^{2+}$ concentrations thereby

73 complicating the measurement. Thus, $\mathrm{Co}^{2+}$ was used to further characterize the transport activity 74 of TsFpn. TsFpn mediates $\mathrm{Co}^{2+}$ uptake and we measured the uptake at different $\mathrm{Co}^{2+}$

75 concentrations. The initial rate of uptake versus ion concentration can be fit with a Michaelis

76 Menten equation with a $\mathrm{K}_{\mathrm{M}}$ of $9.7 \pm 3.26 \mu \mathrm{M}$ and $\mathrm{V}_{\max }$ of $0.20 \pm 0.03 \Delta \mathrm{F} / \mathrm{min}$ (Fig.1d, e). TsFpn 
77 is sensitive to human hepcidin ${ }^{3}$, and the inhibition reaches $\sim 50 \%$ likely due to the random

78 orientation of TsFpn on the liposomes (Fig. 1f). We also measured $\mathrm{Co}^{2+}$ binding to the purified

79 TsFpn using isothermal titration calorimetry (ITC) and found that the binding is exothermic with

$80 \mathrm{a} \Delta \mathrm{H}$ of $-12.0 \pm 0.55 \mathrm{~kJ} / \mathrm{mol}$ and $\mathrm{T} \Delta \mathrm{S}$ of $9.29 \pm 0.38 \mathrm{~kJ} / \mathrm{mol}$. TsFpn binds to $\mathrm{Co}^{2+}$ with a

81 dissociation constant $\left(\mathrm{K}_{\mathrm{d}}\right)$ of $182.6 \pm 16.8 \mu \mathrm{M}$ (Fig. $\left.1 \mathrm{~g}\right)$.

82

\section{Structure of TsFpn}

84

85 We prepared monoclonal antibodies against TsFpn to facilitate its structural determination ${ }^{24}$

86 (Methods). TsFpn forms a stable complex with the antigen binding fragment (Fab) of a

87 monoclonal antibody $11 \mathrm{~F} 9$ as indicated by a shift in the retention time of the elution peak on the

88 size-exclusion column (Extended Data Fig. 1a-b). To assess the effect of 11F9 Fab on TsFpn, we

89 examined $\mathrm{Co}^{2+}$ binding and transport in the presence of the Fab. Fab-TsFpn complex has a

90 modestly reduced affinity to $\mathrm{Co}^{2+}$ with a $\mathrm{K}_{\mathrm{d}}$ of $258.2 \pm 34.2 \mu \mathrm{M}$ (Extended Data Fig. 2a).

91 However, addition of the Fab inhibits $\mathrm{Co}^{2+}$ uptake, and the inhibition reaches $\sim 50 \%$ at $1 \mu \mathrm{M}$ of

92 Fab (Extended Data Fig. 2b-c). These results suggest that the Fab has a modest effect on ion

93 binding and a pronounced effect on ion transport, likely by hindering conformational changes of

94 TsFpn.

96 We reconstituted TsFpn-11F9 into nanodisc (Extended Data Fig.1a) and prepared cryo-EM grids

97 in the presence of $10 \mathrm{mM}$ of $\mathrm{Co}^{2+}$. The images show recognizable particles of TsFpn-11F9

98 complex and we were able to obtain a final map at $3.0 \AA$ A overall resolution (Fig. 2a, Extended

99 Data Figs. 3 and 4 and Extended Data Table 1). The map shows clear density for all 
100

101

102

103

104

105

106

107

108

109

110

111

112

113

114

115

116

117

118

119

120

121

122

transmembrane helices and resolves most of the side chains (Extended Data Fig. 5) to allow de novo building of the TsFpn structure. The final structure model includes residues 17 to 237,289

to 395 and 453 to 552 . The N-terminal 16 residues, two loops between TM6 and 7 and TM9 and 10, and C-terminal 25 residues were not resolved. These regions are predicted to be unstructured (Extended Data Fig. 6). For the Fab fragment, the constant region was not fully resolved and was built as poly alanines while the variable region is well resolved with a local resolution close to $2.9 \AA$ (Extended Data Fig. 3).

TsFpn adopts a canonical MFS fold ${ }^{25}$. The 12 transmembrane helices are packed into two clearly defined domains. TM1-6 form the N-domain, and TM7-12 the C-domain (Fig. 2b-d). Based on previous studies of human Fpn topology ${ }^{26}$ and the "positive-inside" rule ${ }^{27}$, both the N- and Ctermini are located to the cytosolic side. The two domains are connected by a long intracellular loop between TM6 and TM7. Part of the loop is an amphipathic helix that extends horizontally and oriented parallel to the intracellular surface of the membrane (Fig. 2b).

TsFpn structure is in an outward facing conformation. The $\mathrm{N}$ - and $\mathrm{C}$-domains make contact at the cytosolic side. Asp157 on TM4 is in close proximity to Arg88 on TM3 and Arg490 on TM11 and could form salt bridges with the arginines (Fig. 2d). These residues are conserved in the MFS family of transporters and are commonly known as the motif- $\mathrm{A}^{25}$. Other interactions between the $\mathrm{N}$ - and C-domains include Arg178 in the N-domain and Asp474 in the C-domain, and Asn 174 in the $\mathrm{N}$-domain and Gln482 in the C-domain. These interacting pairs of residues are conserved in human Fpn (Extended Data Fig. 6) and mutations to Arg88, Asp157, Asn174, $\operatorname{Arg} 178$, and Arg490 are known to cause ferroportin diseases ${ }^{5,28,29}$. 


\section{Potential metal ion binding sites}

TsFpn structure has a large solvent-accessible central cavity between the N-and C-domains (Fig. arginine residues, the electrostatic surface potential of the cavity is highly negative (Fig. 2e). metal ion binding sites. The first site, S1, is coordinated by Asp39 and His43, and the second site, S2, Cys326 and His508 (Fig. 3a-c). S1 is located in the N-domain while S2 is in the C-domain. are solvent accessible from the extracellular side, and the distance between the two sites are 16.0

$134 \AA$ as measured between the two ions. These two metal ion binding sites are unusual because both

135 the S1 and S2 sites are coordinated by only 2 residues, which is very different from the ion binding sites identified in other transition metal ion transporters of known structures, such as $\mathrm{NRAMP}^{30,31}, \mathrm{VIT}^{31}, \mathrm{YiiP}^{32}, \mathrm{ZIP}^{33}$ and $\mathrm{ZneA}^{34}$, all of which have at least four residues coordinating a metal ion binding site. In addition, S2 does not have a charged residue making

139 direct contact with the bound ion, although two negatively charge residues, Asp325 and Asp505

140 are located close to S2 and could potentially interact with Cys326 and His508, respectively. It is

141 also unusual to have two substrate binding sites because most other members of the MFS family

142 of transporters have a single substrate binding site often coordinated by residues from both the

$143 \mathrm{~N}$ - and C-domains ${ }^{13-19}$. To further understand how S1 and S2 may participate in ion transport, we 144 examined the binding sites with functional studies. 


\section{TsFpn is an electroneutral $\mathrm{H}^{+} / \mathrm{Fe}^{2+}$ antiporter}

147

148

As a first test to validate the ion binding sites, we examined $\mathrm{pH}$ dependency of metal ion binding and transport in TsFpn because both the $\mathrm{S} 1$ and $\mathrm{S} 2$ sites contain a histidine. TsFpn does not bind to $\mathrm{Co}^{2+}$ in $\mathrm{pH} 6.0$, and the binding affinity gradually recovers as $\mathrm{pH}$ increases from 6.0 to 8.0 (Extended Data Figs.1c and 7a-f). Similarly, $\mathrm{Co}^{2+}$ uptake is significantly reduced when external $\mathrm{pH}$ is 6.5, and the uptake gradually increases as external $\mathrm{pH}$ increases from 6.5 to 8.5 (Fig. 4a). These results are consistent with the presence of histidine residues at the ion binding site and provide the first systematic evaluation of $\mathrm{pH}$ dependency of ion binding and transport in Fpn.

Intrigued by the drastically enhanced $\mathrm{Co}^{2+}$ uptake at elevated $\mathrm{pH}(8.0$ and 8.5$)$, we wondered whether it was the lower $\mathrm{H}^{+}$concentration or the $\mathrm{H}^{+}$gradient responsible for enhanced metal ion transport. Ion transport under symmetrical $\mathrm{pH} 8.0$ or 8.5 is not significantly different from that in symmetrical pH 7.5 (Fig. 4b), indicating that metal ion uptake is enhanced by the $\mathrm{pH}$ gradient, i.e., a higher $\mathrm{H}^{+}$concentration inside of the liposomes. These results suggest that TsFpn is a $\mathrm{H}^{+} / \mathrm{Fe}^{2+}$ antiporter in which metal ion transport is coupled to $\mathrm{H}^{+}$movement in the opposite direction. We further tested this hypothesis in the following three experiments.

We first varied salt composition in the assay buffer and we found that neither $\mathrm{Na}^{+}, \mathrm{K}^{+}$nor $\mathrm{Cl}^{-}$ enhances metal ion transport (Fig. 4c). Second, we measured proton transport directly in a flux assay. In this experiment, uptake of $\mathrm{H}^{+}$is monitored by a proton sensitive fluorescent dye pyranine trapped inside of the liposomes and the $\mathrm{H}^{+}$uptake is driven by efflux of $\mathrm{Co}^{2+}$ (Fig. 4d-e and Methods). This result confirms the coupled movement of $\mathrm{H}^{+}$and $\mathrm{Co}^{2+}$ in opposite directions. 
169 Third, we determined the stoichiometry of the coupled $\mathrm{H}^{+}$and $\mathrm{Co}^{2+}$ movement by examining if 170 metal ion transport in TsFpn is affected by a membrane potential. We set the membrane potential

171 at $\sim 120 \mathrm{mV}$ by having a 100 -fold $\mathrm{K}^{+}$concentration gradient in the presence of a $\mathrm{K}^{+}$selective

172 ionophore valinomycin. Membrane potential of a vesicle is defined assuming $0 \mathrm{mV}$ at outside.

173 We found that the membrane potential has no effect on the rate of either $\mathrm{Fe}^{2+}$ or $\mathrm{Co}^{2+}$ uptake (Fig.

174 4f-g). This result indicates that metal ion transport in TsFpn is electroneutral and that the most

175 likely stoichiometry of $\mathrm{H}^{+}$to $\mathrm{Fe}^{2+}$ is 2 to 1 . Taken together, our results led to the conclusion that

176 TsFpn is an electroneutral $2 \mathrm{H}^{+} / \mathrm{Fe}^{2+}$ antiporter.

177

Mutational study of metal ion binding sites

As a second test to validate the ion binding sites, we made mutations to the $\mathrm{S} 1$ and $\mathrm{S} 2$ binding

181 sites and measured ion binding and transport. Two double mutations were made: the S1 mutation, i.e., Asp39Ala-His43Ala, and the S2 mutation, i.e., Cys326Ala-His508Ala. Both the S1 and S2 mutations can be purified and are stable after purification (Extended Data Fig. 1d-e). We measured metal ion transport in symmetrical $\mathrm{pH} 7.5$ and with a $\mathrm{pH}$ gradient $(\mathrm{pH} 7.5$ inside and $\mathrm{pH} 8.5$ outside), and we determined if ion transport remains electroneutral.

187 The S1 mutation has similar transport activity to that of the wild type TsFpn in symmetrical $\mathrm{pH}$ increase is much smaller than the increase observed in the wild type (Fig. $4 \mathrm{~b}$ and $4 \mathrm{~h}$ ). This result indicates that the coupled transport of $\mathrm{H}^{+}$and $\mathrm{Co}^{2+}$ is affected by the $\mathrm{S} 1$ mutation. Consistent with this conclusion, $\mathrm{Co}^{2+}$ transport in the $\mathrm{S} 1$ mutation becomes electrogenic and shows a large 
192 increase in transport activity under a membrane potential of $\sim 120 \mathrm{mV}$ (Fig. 4i). Enhanced $\mathrm{Co}^{2+}$

193 transport under $-120 \mathrm{mV}$ indicates that less than two $\mathrm{H}^{+}$is transported for each $\mathrm{Co}^{2+}$, i.e., $\mathrm{H}^{+}$

194 transport is impaired. Since $\mathrm{Co}^{2+}$ transport is about similar to that of the wild type in symmetrical

$195 \mathrm{pH}$, it is likely the $\mathrm{S} 1$ mutation affects $\mathrm{H}^{+}$transport.

197 The S2 mutation has significantly lower transport activity than that of the wild type under both 198 the symmetrical and $\mathrm{pH}$ gradient conditions. Transport activity is enhanced in the $\mathrm{pH}$ gradient, 199 although it is difficult to determine if the enhancement is similar to that of the wild type (Fig. 4h). $200 \mathrm{Co}^{2+}$ transport is also enhanced under $-120 \mathrm{mV}$ membrane potential, indicating that less than 2 $201 \mathrm{H}^{+}$is transported for each $\mathrm{Co}^{2+}$ (Fig. 4i). Since $\mathrm{Co}^{2+}$ transport is significantly lower in 202 symmetrical $\mathrm{pH}$, it is likely the $\mathrm{S} 2$ mutation affects both $\mathrm{H}^{+}$and $\mathrm{Co}^{2+}$ transport.

Both S1 and S2 mutations can still transport proton and the effects of the mutations mirror the 205 effects observed in the $\mathrm{Co}^{2+}$ transport assay (Fig.4j). Combined, these results led us to conclude 206 that both the S1 and S2 sites are important for $\mathrm{H}^{+}$transport, while for $\mathrm{Co}^{2+}$ transport the $\mathrm{S} 2$ site is 207 critical and the S1 site seems redundant. Because the S2 mutation maintains a small yet 208 significant level of transport, it is likely that the S1 site could mediate metal ion transport but not 209 as efficiently.

211 To estimate how the two sites contribute to metal ion binding, we measured $\mathrm{Co}^{2+}$ binding to the $212 \mathrm{~S} 1$ and $\mathrm{S} 2$ mutations by ITC. The $\mathrm{S} 1$ mutation binds to $\mathrm{Co}^{2+}$ with a $\mathrm{K}_{\mathrm{d}}$ of $266.3 \pm 23.8 \mu \mathrm{M}$, and 213 the S2 mutation binds to $\mathrm{Co}^{2+}$ with a $\mathrm{K}_{\mathrm{d}}$ of $162.4 \pm 16.0 \mu \mathrm{M}$ (Extended Data Fig. 7g-h). When 214 both sites are mutated (Asp39Ala/His43Ala/Cys326Ala/His508Ala), the binding affinity 
215 becomes $616.0 \pm 44.9 \mu \mathrm{M}$ (Extended Data Figs. 1f and 7i). These results suggest that both S1

216 and $\mathrm{S} 2$ site contribute to metal ion binding.

\section{Discussion}

In summary, we solved the structure of TsFpn in an outward-facing conformation and we

221 identified two potential metal ion binding sites $\mathrm{S} 1$ and $\mathrm{S} 2$. We also found that TsFpn is a $\mathrm{H}^{+} / \mathrm{Fe}^{2+}$

222 antiporter and the transport of each $\mathrm{Fe}^{2+}$ is coupled to two $\mathrm{H}^{+}$. Further studies showed that both

$223 \mathrm{~S} 1$ and $\mathrm{S} 2$ sites are involved in $\mathrm{H}^{+}$transport while the $\mathrm{S} 2$ site is more critical to metal ion

224 transport.

225

226 We generated a model of TsFpn in the inward facing conformation by aligning the $\mathrm{N}$ - and $\mathrm{C}$ -

227 domains separately on their equivalent domains in the inward-open bacterial BbFpn structure ${ }^{21}$

228 (Extended Data Fig. 8a). The transmembrane domains of the two structures align reasonably well

229 with a root mean square distance of $3.6 \AA$ for the $\mathrm{N}$-domain and that $2.1 \AA$ for the C-domain.

230 Both the S1 and S2 sites are solvent accessible in the inward-facing model of TsFpn (Extended

231 Data Fig. 8b), suggesting that a canonical rock-switch type motion of the N-and C-domains

232 could achieve alternating access to the substrates (Fig.5a-d). We speculate that two $\mathrm{H}^{+}$could

233 bind to both $\mathrm{S} 1$ and $\mathrm{S} 2$ and be transported across the membrane, while one $\mathrm{Fe}^{2+}$ could bind to

234 either S1 or S2 sites and be transported in the opposite direction. Further study is required to

235 reveal details of how structural changes during the transport allow the two sites to coordinate and

236 transport $\mathrm{H}^{+}$and $\mathrm{Fe}^{2+}$ in opposite directions. 
Although TsFpn and BbFpn share the same MFS fold, the S1 and S2 binding sites in TsFpn differ from the ion binding sites identified in BbFpn. The metal ion binding site identified in the initial BbFpn structure is formed by residues Thr20, Asp24, Asn196, Ser199, and Phe200, and these residues correspond to Ser35, Asp39, Asn212, Ser215, and Met216 in TsFpn (Extended Data Figs. 6 and 9a-b). However, only Asp39 is involved in S1 and when the two structures are aligned, the two binding sites are $8.6 \AA$ away (Extended Data Fig. 9a). In a more recent publication, another metal ion binding site was identified in the BbFpn formed by residues Trp254, His261 and Thr386. However, the new metal ion binding site contains an EDTA molecule that helps coordinate a bound metal ion $^{22}$. This site is close to $\mathrm{S} 2$ in TsFpn, however, none of the coordinating residues are part of the S2 (Extended Data Figs. 6 and 9c). Bacterial BbFpn was also shown to have higher rate of uptake when external $\mathrm{pH}$ increases, but the enhanced transport was due to the $\mathrm{pH}$ and not the $\mathrm{pH}$ gradient ${ }^{21}$. This result indicates that $\mathrm{BbFpn}$ has a different metal ion transport mechanism.

We mapped known missense mutations that cause ferroportin diseases onto the TsFpn structure (Fig. 5e). The structure provides insights into how certain mutations may lead to diseases. For example, six mutations are mapped to regions close to S1 or S2 and these mutations likely affect ion binding. Eighteen mutations are mapped to regions where the $\mathrm{N}$ - and $\mathrm{C}$-domain make contact in the current structure and these mutations likely affect transport activity. Moreover, we show that transport activity of TsFpn is inhibited by human hepcidin, and we mapped residues known to affect hepcidin inhibition to the TsFpn structure ${ }^{3}$. These residues cluster mostly on the extracellular surface with a few deeper into the cavity including Cys326 of the S2 site. It appears that hepcidin could perturb either $\mathrm{H}^{+}$or $\mathrm{Fe}^{2+}$ binding by interacting with the $\mathrm{S} 2$ site. 


\section{Methods}

\section{Cloning, expression, and purification of TsFpn}

264 The Fpn gene (accession number XP_008060857) from Carlito syrichta (Tarsius syrichta,

265 Philippine tarsier) was codon-optimized and cloned into a pFastBac dual vector ${ }^{32}$ for production

266 of baculovirus by the Bac-to-Bac method (Invitrogen). High Five Cells (Thermofisher) at a

267 density of $\sim 3 \times 10^{6}$ cells/ml were infected with baculovirus and grown at $27^{\circ} \mathrm{C}$ for $60-70$ hour

268 before harvesting. Cell membranes were prepared following a previous protocol ${ }^{32}$ and frozen in 269 liquid nitrogen.

270 Purified membranes were thawed and homogenized in $20 \mathrm{mM}$ HEPES, $\mathrm{pH}$ 7.5, $150 \mathrm{mM} \mathrm{NaCl}$ 271 and $2 \mathrm{mM} \beta$-mercaptoethanol, and then solubilized with $1 \%(\mathrm{w} / \mathrm{v})$ Lauryl Maltose Neopentyl

272 Glycol (LMNG, Anatrace) at $4{ }^{\circ} \mathrm{C}$ for $2 \mathrm{~h}$. After centrifugation $\left(55,000 \mathrm{~g}, 45 \mathrm{~min}, 4{ }^{\circ} \mathrm{C}\right)$, TsFpn 273 was purified from the supernatant using a cobalt-based affinity resin (Talon, Clontech) and the

274 His $_{6}$-tag was cleaved by TEV protease at room temperature for 1 hour. TsFpn was then

275 concentrated to 3-6 mg/ml (Amicon $50 \mathrm{kDa}$ cutoff, Millipore) and loaded onto a size-exclusion

276 column (SRT-3C SEC-300, Sepax Technologies, Inc.) equilibrated with $20 \mathrm{mM}$ HEPES, pH7.5,

$277150 \mathrm{mM} \mathrm{NaCl}, 1 \mathrm{mM}(\mathrm{w} / \mathrm{v})$ n-dodecyl- $\beta$-D-maltoside (DDM, Anatrace).

278

279 Mutations to TsFpn were generated using the QuikChange method (Stratagene) and the entire 280 cDNA was sequenced to verify the mutation. Mutants were expressed and purified following the 281 same protocol as wild type. 
284 Monoclonal antibodies against the TsFpn $(\operatorname{IgG} 2 \mathrm{~b}, \kappa)$ were raised using standard methods

285 (Monoclonal Core, Vaccine and Gene Therapy Institute, Oregon Health \& Science University).

286 High affinity and specificity of the antibodies for properly folded TsFpn was assayed by ELISA

287 and western blot (no binding). Three out of twenty antibodies were selected for large scale

288 production. Fab fragments were generated by papain cleavage of whole antibody at a final

289 concentration of $1 \mathrm{mg} / \mathrm{mL}$ for 2 hours at $37^{\circ} \mathrm{C}$ in $50 \mathrm{mM}$ Phosphate buffer saline, pH 7.0, $1 \mathrm{mM}$

290 EDTA, $10 \mathrm{mM}$ cysteine and 1:50 w:w papain:antibody. Digestion was quenched using $30 \mathrm{mM}$

291 iodoacetamide at $25^{\circ} \mathrm{C}$ for $10 \mathrm{~min}$. Fab was purified by anion exchange using a Q Sepharose

292 (GE Healthcare) column in $10 \mathrm{mM}$ Tris, $\mathrm{pH} 8.0$ and a $\mathrm{NaCl}$ gradient elution. TsFpn-Fab

293 complexes were further verified by size-exclusion chromatography (shift in elution volume and

294 SDS-PAGE) and 11F9 was selected for structural studies.

\section{TsFpn-11f9(Fab) complex}

297 Purified TsFpn was mixed with the 11F9 Fab at 1:1.1 molar ratio and incubated 30 min on ice.

298 The complex was then concentrated to 3-6 mg/ml (Amicon $100 \mathrm{kDa}$ cutoff, Millipore) and

299 loaded onto a size-exclusion column equilibrated with $20 \mathrm{mM}$ HEPES, pH7.5, $150 \mathrm{mM} \mathrm{NaCl}, 1$ mM n-dodecyl- $\beta$-D-maltoside (DDM, Anatrace). The TsFpn-Fab complex was used in the ITC measurement of $\mathrm{Co}^{2+}$ binding and in nanodisc reconstitution for cryo-EM grid preparations.

\section{Nanodisc reconstitution}

304 MSP1D1 was expressed and purified following an established protocol ${ }^{33}$. For lipid preparation,

305 1-palmitoyl-2-oleoyl-sn-glycero-3-phospho-(1'-rac)-choline (POPC, Avanti Polar Lipids), 1-

306 palmitoyl-2-oleoyl-sn-glycero-3-phospho-(1'-rac)-ethanolamine (POPE, Avanti Polar Lipids) 
and 1-palmitoyl-2-oleoyl-sn-glycero-3-phospho-(1'-rac)-glycerol (POPG, Avanti Polar Lipids) were mixed at a molar ratio of 3:1:1, dried under Argon and resuspended with 14 mM DDM ${ }^{34}$. For nanodisc reconstitution, TsFpn, Fab of 11f9, MSP1D1 and lipid mixture were mixed at a molar ratio of 1:(1.1):(2.5):(62.5) and incubated on ice for 1 hour. Detergents were removed by incubation with Biobeads SM2 (Bio-Rad) overnight at $4{ }^{\circ} \mathrm{C}$. The protein lipid mixture was loaded onto a size-exclusion column equilibrated with $20 \mathrm{mM}$ HEPES, pH7.5, $150 \mathrm{mM} \mathrm{NaCl}$. The purified nanodisc elutes at $13.6 \mathrm{ml}$ and was concentrated to $13 \mathrm{mg} / \mathrm{ml}$ and incubated with 10 $\mathrm{mM} \mathrm{CoCl} 2$ for 30 min before cryo-EM grid preparation.

\section{Cryo-EM sample preparation and data collection}

The cryo grids were prepared using Thermo Fisher Vitrobot Mark IV. The Quantifoil R1.2/1.3

$\mathrm{Cu}$ grids were glow-discharged with air for $15 \mathrm{sec}$ at $10 \mathrm{mM}$ in a Plasma Cleaner (PELCO EasiGlow $^{\mathrm{TM}}$ ). Aliquots of $3.5 \mu \mathrm{l}$ purified TsFpn-11f9 in nanodisc were applied to glowdischarged grids. After being blotted with filter paper (Ted Pella, Inc.) for $4.0 \mathrm{~s}$, the grids were plunged into liquid ethane cooled with liquid nitrogen. A total of 1838 micrograph stacks were collected with SerialEM ${ }^{35}$ on a Titan Krios at $300 \mathrm{kV}$ equipped with a K2 Summit direct electron detector (Gatan), a Quantum energy filter (Gatan) and a Cs corrector (Thermo Fisher), at a nominal magnification of $105,000 \times$ and defocus values from $-2.0 \mu \mathrm{m}$ to $-1.2 \mu \mathrm{m}$. Each stack was exposed in the super-resolution mode for $5.6 \mathrm{~s}$ with an exposing time of $0.175 \mathrm{~s}$ per frame, resulting in 32 frames per stack. The total dose rate was about $50 \mathrm{e}^{-} / \AA^{2}$ for each stack. The stacks were motion corrected with MotionCor $2^{36}$ and binned 2 fold, resulting in a pixel size of 1.114 $\AA /$ pixel. In the meantime, dose weighting was performed ${ }^{37}$. The defocus values were estimated with $\mathrm{Gctf}^{38}$. 


\section{Cryo-EM data processing}

332 A total of 1,246,999 particles were automatically picked with RELION 2.1 ${ }^{39-41}$. After 2D

334 classification with one class and 40 iterations. The outputs of the 35 th- 40 th iterations were

335 subjected to local angular search 3D classification with four classes separately. A total of

571,511 particles were selected by combining the good classes of the local angular search 3D

337 classification. After handedness correction, a skip-align classification procedure was performed

The resolution of the map was further improved to $3.0 \AA$ after Bayesian polishing ${ }^{42}$.

All 2D classification, 3D classification, and 3D auto-refinement were performed with RELION

2.1 or RELION 3.0. Resolutions were estimated with the gold-standard Fourier shell correlation

\section{Model building and refinement}

347 For de novo model building of TsFpn-11F9 complex, a ploy-Alanine model was first manually

349 refinements were carried out by PHENIX in real space with secondary structure and geometry

350 restraints $^{46}$. The EMRinger Score was calculated as described ${ }^{47}$.

\section{Proteoliposome preparation}


353 POPE and POPG (Avanti Polar Lipids) was mixed at 3:1 molar ratio, dried under Argon and

354 vacuumed overnight to remove chloroform. The lipid was resuspended in the reconstitution

355 buffer (20 mM HEPES, pH 7.5, $100 \mathrm{mM} \mathrm{NaCl})$ to a final concentration of $10 \mathrm{mg} / \mathrm{ml}$, sonicated

356 to transparency and incubated with $40 \mathrm{mM}$-decyl- $\beta$-D-maltoside (DM, Anatrace) for $2 \mathrm{~h}$ at

357 room temperature under gentle agitation. Wild type or mutant TsFpn was added at 1:100 (w/w,

358 protein:lipid) ratio. The detergent was removed by dialysis at $4{ }^{\circ} \mathrm{C}$ against the reconstitution

359 buffer. Dialysis buffer was changed once a day and the liposomes were harvested after 4 days,

360 aliquoted, and frozen at $-80{ }^{\circ} \mathrm{C}$.

361

Divalent metal ion flux assay

363 Liposomes were mixed with $250 \mu \mathrm{M}$ calcein and frozen-thawed three times. After the liposomes

364 were extruded to homogeneity with $400 \mathrm{~nm}$ filter (NanoSizer ${ }^{\mathrm{TM}}$ Extruder, T\&T Scientific

365 Corporation), free calcein was removed through a desalting column (PD-10, GE Healthcare)

366 equilibrated with the dialysis buffer. Calcein fluorescence was monitored in a quartz cuvette at

$36737^{\circ} \mathrm{C}$. Fluorescence was monitored in a FluoroMax-4 spectrofluorometer (HORIBA) with 494

$368 \mathrm{~nm}$ excitation and $513 \mathrm{~nm}$ emission at $10 \mathrm{~s}$ internals. The transport was initiated by the addition

369 of $0.5 \mathrm{mM} \mathrm{CoCl} 2$ or $100 \mu \mathrm{M}$ fresh prepared $\mathrm{FeSO}_{4}$. When $\mathrm{Fe}^{2+}$ is used, $1 \mathrm{mM}$ vitamin $\mathrm{C}$ was

370 added in the external solution. The rate of ion transport is estimated by the initial slope of the

371 traces of fluorescence quench.

372

373 In experiments when internal solution needs to be replaced, liposomes were centrifuged at

$37447000 \mathrm{~g}$ for $30 \mathrm{~min}$ and resuspended in a desired internal solution. A fluorescent dye was then 
375 loaded into the liposomes by the same freeze-thaw processes and free dye was removed by a

376 desalting column.

\section{Pyranine assay}

379 Liposomes were centrifuged at $47000 \mathrm{~g}$ for $30 \mathrm{~min}$ and resuspended in inside buffer (5 mM Tris, $380 \mathrm{pH} 8.5,100 \mathrm{mM} \mathrm{NaCl}$ ). Liposomes were mixed with $250 \mu \mathrm{M}$ pyranine and $2 \mathrm{mM} \mathrm{CoCl}_{2}$ and 381 frozen-thawed three times. After the liposomes were extruded to homogeneity with $400 \mathrm{~nm}$ filter 382 (NanoSizer ${ }^{\mathrm{TM}}$ Extruder, T\&T Scientific Corporation), free dye was removed through a desalting column (PD-10, GE Healthcare) equilibrated with the outside buffer (5 mM HEPES, pH 7.5, 100 $\mathrm{mM} \mathrm{NaCl}, 2 \mathrm{mM} \mathrm{CoCl}_{2}$ ). Pyranine fluorescence was monitored in a quartz cuvette at $37^{\circ} \mathrm{C}$ in a

385 FluoroMax-4 spectrofluorometer (HORIBA) with $460 \mathrm{~nm}$ excitation and $510 \mathrm{~nm}$ emission at 10 $386 \mathrm{~s}$ internals. The transport was initiated by the addition of $2 \mathrm{mM}$ EDTA.

\section{Isothermal titration calorimetry}

Protein samples were purified as described above and concentrated to around 50-100 $\mu \mathrm{M}(3-6$ $\mathrm{mg} / \mathrm{ml})$. TsFpn was in the ITC buffer that contains $20 \mathrm{mM}$ HEPES pH 7.5, $150 \mathrm{mM} \mathrm{NaCl,} 1 \mathrm{mM}$

394 injections. The background data obtained from injecting $\mathrm{Co}^{2+}$ into the ITC buffer were subtracted 395 before the data analysis. The data were fitted using the Origin8 software package (MicroCal). 396 Measurements were repeated three times. 


\section{Acknowledgments}

399 This work was supported by grants from NIH (DK122784, HL086392 and GM098878 to MZ),

400 Cancer Prevention and Research Institute of Texas (R1223 to MZ). Ara Parseghian Medical

401 Research Foundation (to N.Y.). N.Y. is supported by the Shirley M. Tilghman endowed

402 professorship from Princeton University. We thank Paul Shao for technical support during EM

403 image acquisition. We acknowledge the use of Princeton's Imaging and Analysis Center, which

404 is partially supported by the Princeton Center for Complex Materials, and the National Science

405 Foundation (NSF)-MRSEC program (DMR-1420541).

406

407

\section{Data Availability}

408 The atomic coordinates of TsFpn-Fab complex have been deposited in the PDB

409 (http://www.rcsb.org) under the accession codes 6VYH. The corresponding electron microscopy

410 maps have been deposited in the Electron Microscopy Data Bank

411 (https://www.ebi.ac.uk/pdbe/emdb/) under the accession codes EMD-21460.

412

\section{Author Contributions}

414 M.Z., Z.R., Y.P., and J.S. conceived the project. S.G. led the effort of cryo-EM grid

415 preparation, data collection and analysis and was assisted by Z.R., J.S. and L.W.. Y.P., Z.R.,

416 J.S., L.W., Y.Y., H.Z., Z.X., P.B. and A.L. conducted experiments. Y.P., Z.R., G.S., J.S., Z.X.,

417 P.B., A.L., N.Y. and M.Z. analyzed data. Z.R., J.S., Y.P. and M.Z. wrote the initial draft and

418 all authors participated in revising the manuscript.

419

420

\section{Competing interests}


bioRxiv preprint doi: https://doi.org/10.1101/2020.03.04.975748; this version posted March 5, 2020. The copyright holder for this preprint (which was not certified by peer review) is the author/funder, who has granted bioRxiv a license to display the preprint in perpetuity. It is made available under aCC-BY-NC-ND 4.0 International license.

421 The authors declare no competing financial interests.

422

\section{Corresponding authors}

424 Correspondence to Ming Zhou (mzhou@bcm.edu) and Yaping Pan (yaping.pan@bcm.edu).

425

426 

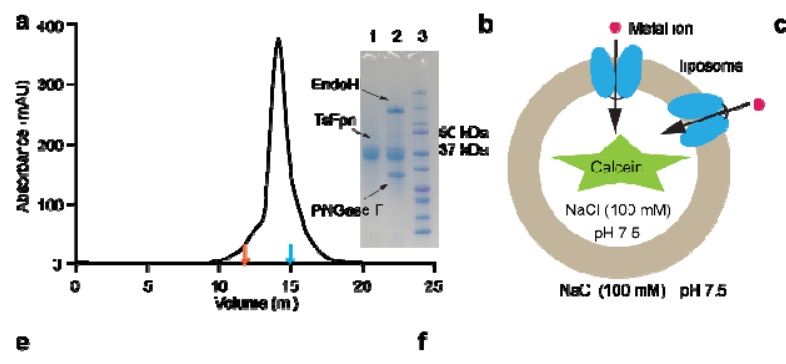

e
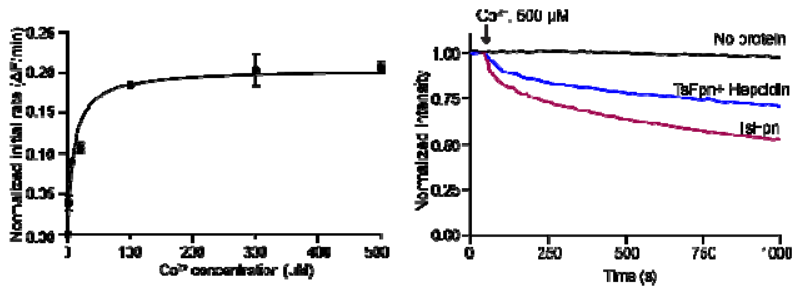

429

Figure 1. Function of purified TsFpn. a. Size-exclusion chromatography profile of TsFpn in detergent DDM. Elution volumes of membrane proteins of known molecular weight, bcMalT $(100 \mathrm{kDa} \text {, orange })^{48}$ and mouse SCD1 (41 kDa, blue) $)^{32}$ are marked by arrows. Inset: SDS-PAGE of purified TsFpn (Lane 1), purified TsFpn with EndoH glycosidase (Lane 2), and standard molecular weight marker (Lane 3). Molecular weights of two the markers are labeled on the right. b. Schematic view of a TsFpn containing proteoliposome with calcein dye enclosed. Influx of metal ions $\left(\mathrm{Fe}^{2+}\right.$ or $\mathrm{Co}^{2+}$, red spheres) quenches the fluorescence. Two orientations of TsFpn are shown. c. Quench of fluorescence in the presence of various concentrations of external $\mathrm{Fe}^{2+}$ at the presence of $1 \mathrm{mM}$ vitamin $\mathrm{C}$ for liposomes with no protein (black line) or liposomes with TsFpn. d. Quench of fluorescence in the presence of different concentrations of external $\mathrm{Co}^{2+}$. e. Initial rate of fluorescence quench versus concentration of $\mathrm{Co}^{2+}$. The solid line is the data fit to a Michaelis Menten equation. f. Quench of fluorescence in the presence of $500 \mu \mathrm{M} \mathrm{Co}{ }^{2+}$ for liposomes with no protein (black line), proteoliposomes with TsFpn (red line), or proteoliposomes with TsFpn and with $1 \mu \mathrm{M}$ hepcidin added to the external side of the liposomes 
444 (blue line). g. ITC measurement of $\mathrm{Co}^{2+}$ binding to TsFpn. Each spike is rate of heat release

445 (upper panel) and each point is the integration of the spike (lower panel). The solid line in the

446 lower panel is fit of the data to a single binding site. Each data point in $\mathbf{e}$ is the average of 3 or

447 more measurements, and the error bars are standard error of the mean (s.e.m.). 
a
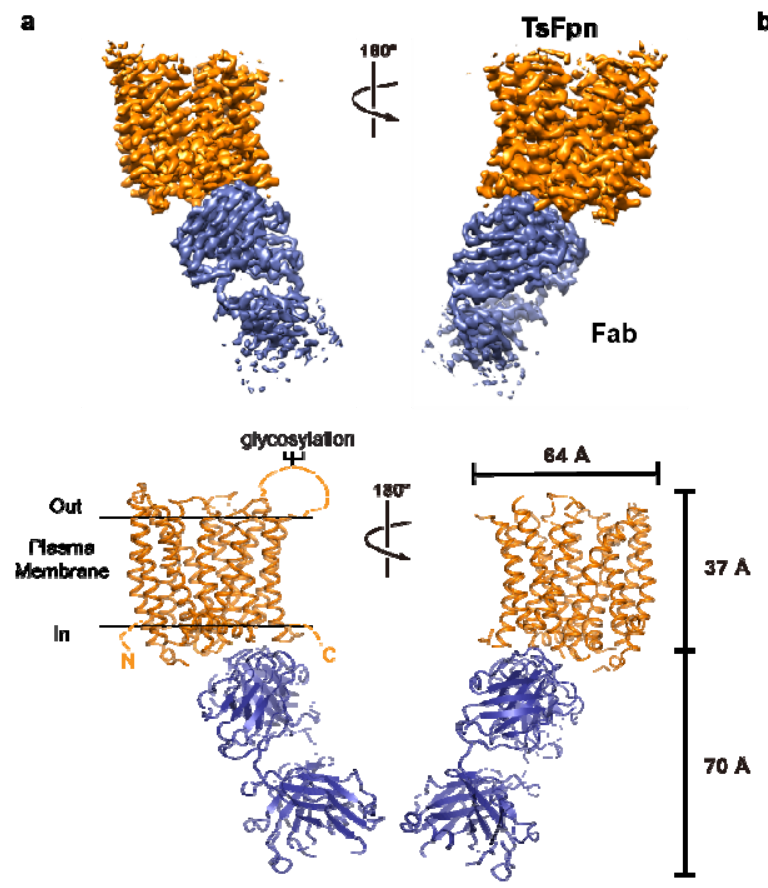

d N-dorain C-domain $\quad$

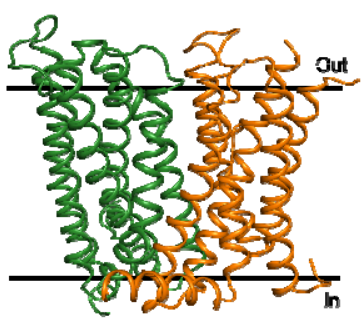

$\frac{1}{V}-\infty$

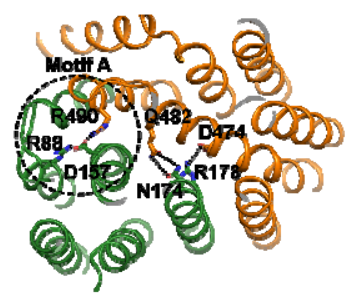

450

451

452

453 b
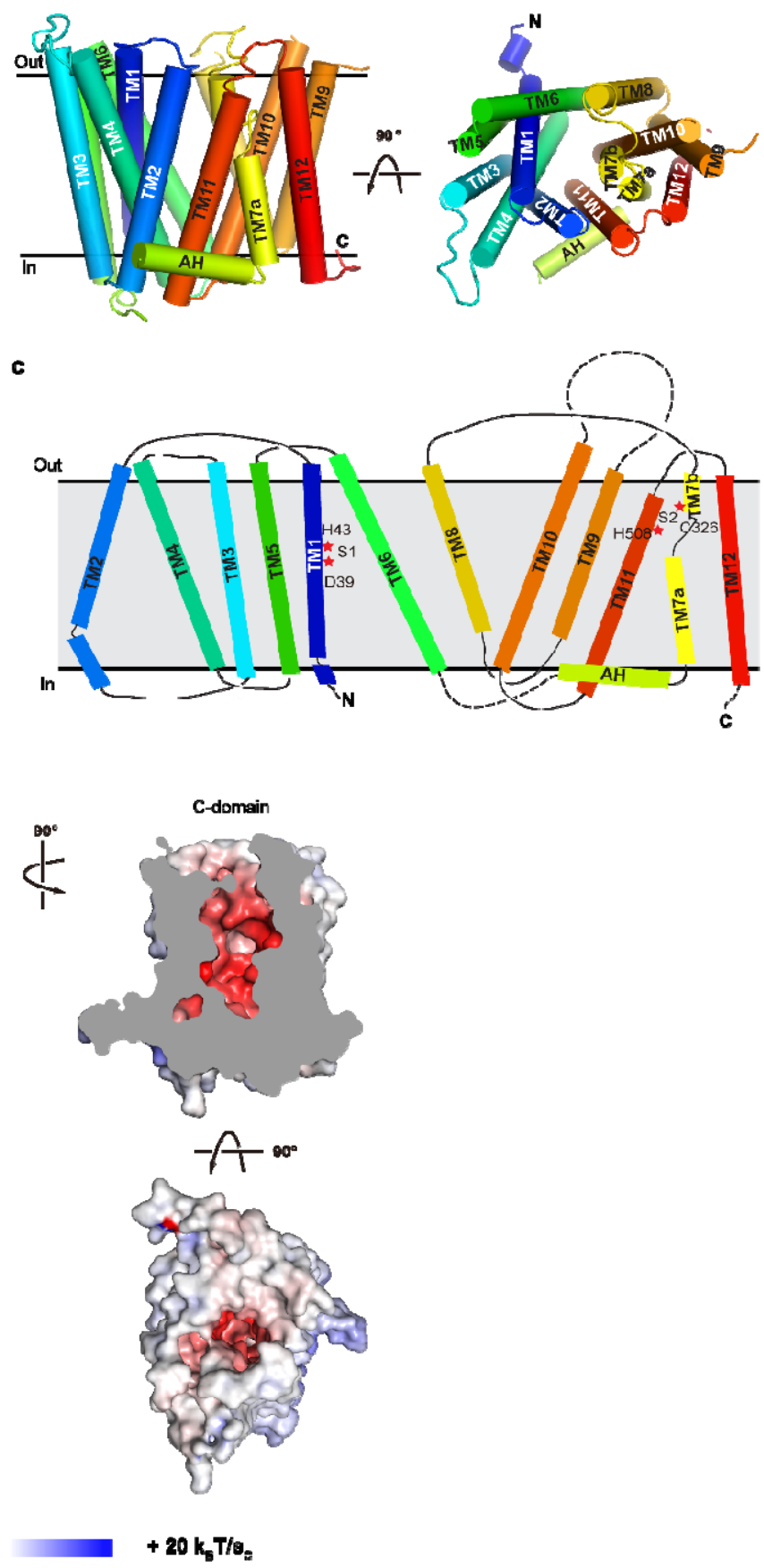

Figure 2. Structure of TsFpn. a. Top panel: cryo-EM map of TsFpn (orange) in complex with Fab (blue) in two views. Bottom panel: TsFpn in complex with Fab shown as ribbon representation. b. TsFpn structure shown as cylinder representation and in two views. c. topology 
454 of TsFpn. Regions that are not resolved in the structure are marked as dotted lines. d. Top: The

$455 \mathrm{~N}$ - and C-domains of TsFpn shown in green and orange, respectively. Bottom: TsFpn viewed

456 from the intracellular side. Interacting residues from the $\mathrm{N}$ - and C-domains are marked as sticks.

457 e. Electrostatic static potential of TsFpn mapped onto the surface representation. The cut-away

458 views show the large cavity formed between the $\mathrm{N}$ - and C-domains. Electrostatic static potential

459 is calculated by $\mathrm{APBS}^{49}$ in Pymol. 
a

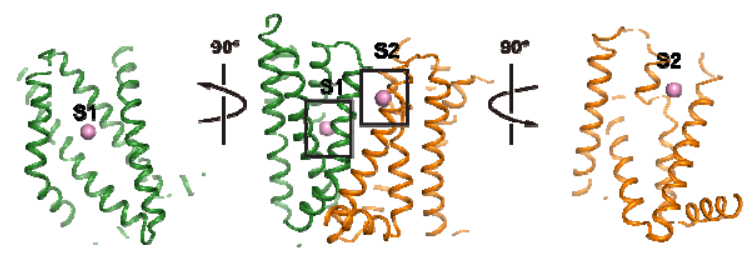

b
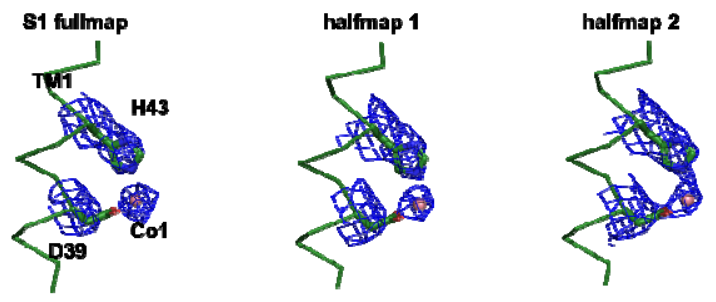

$\mathbf{C}$

2 fullmap

halfmap 1

halfmap 2

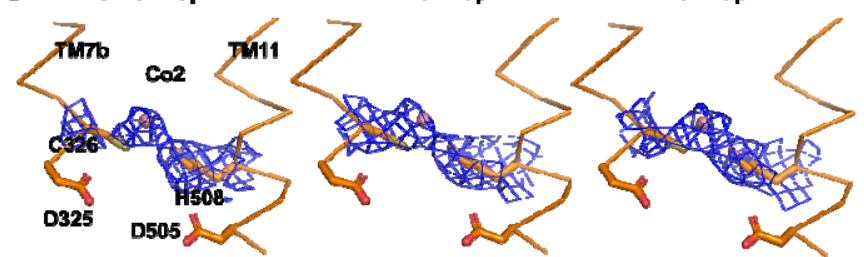

462 Figure 3. Two ion binding sites in TsFpn. a. TsFpn in cartoon representation is shown in three

463 orientations with S1 and S2 marked as sticks. b. Density maps of S1 are shown in blue mesh.

464 Part of the TM1 is shown as trace and the side chains of Asp39 and His43 are shown in stick.

$465 \mathrm{Co}^{2+}$ is shown as a magenta sphere. c. Density maps of S2 are shown in blue mesh. Part of the

466 TM7 and 11 are shown as trace and the side chains of Asp325, Cys326, Asp505 and His508 are

467 shown as sticks. 


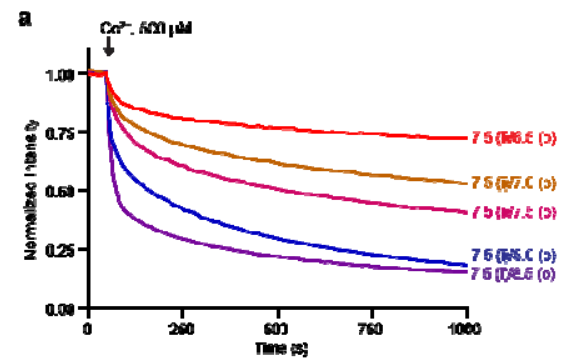

d

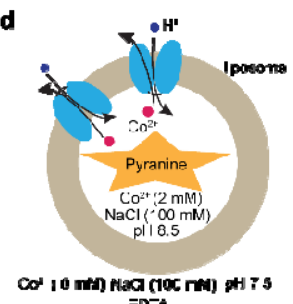

$\equiv D^{-A}$

h

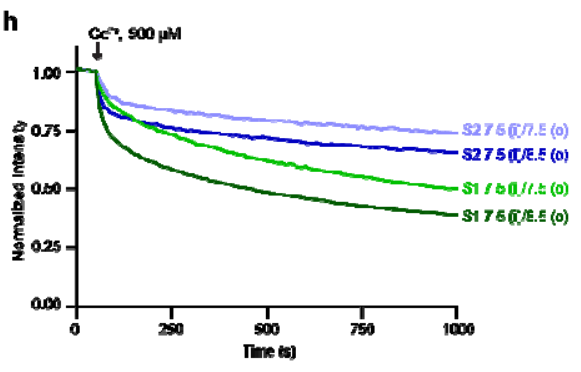

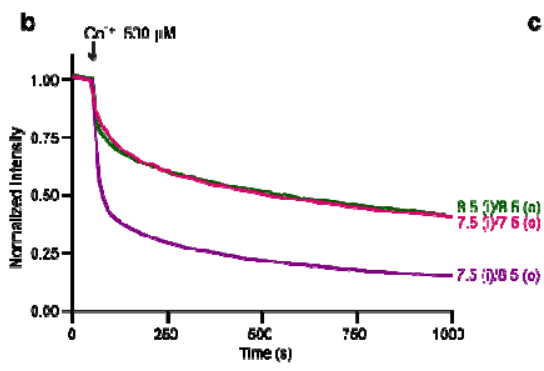

$\mathbf{f}$

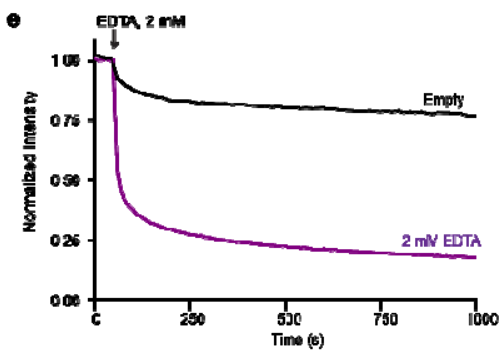

i

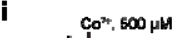

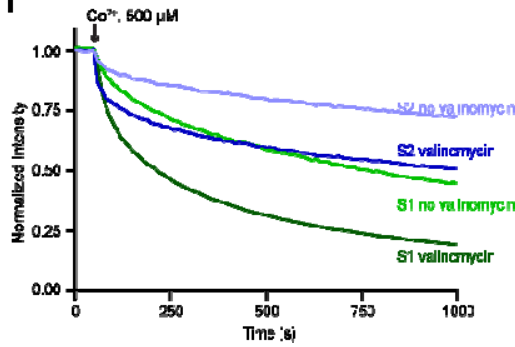

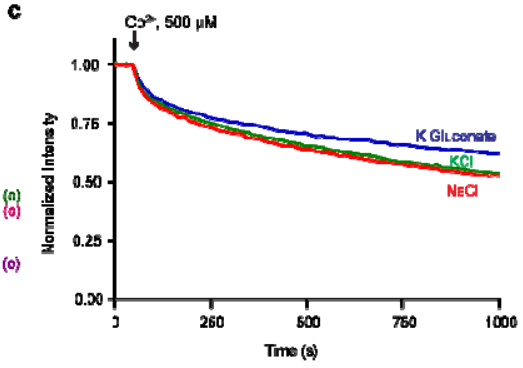

g

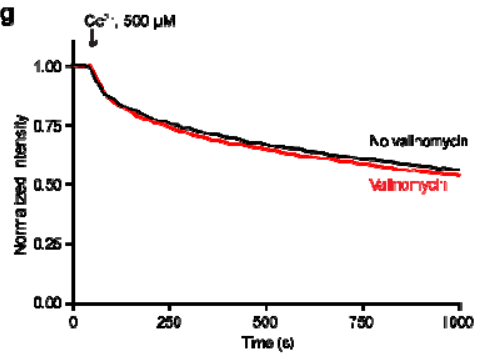

j

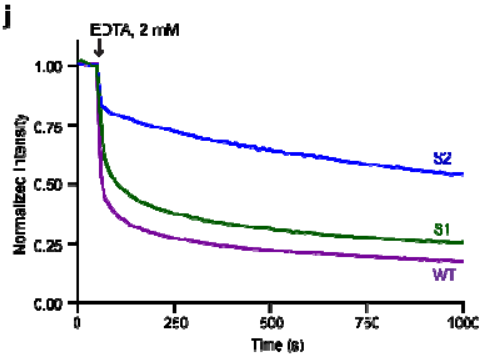

Figure 4. TsFpn is a $\mathrm{H}^{+} / \mathrm{Fe}^{2+}$ antiporter. a. Quench of calcein fluorescence over time measured

471 in different external $\mathrm{pH}$ from 6.5 to 8.5 . The internal $\mathrm{pH}$ is 7.5. b. Quench of calcein

472 fluorescence over time measured in symmetrical $\mathrm{pH}, 7.5$ inside (i) and 8.5 outside (o). The trace

473 for symmetrical $\mathrm{pH} 7.5$ and the trace for $\mathrm{pH} 7.5$ (internal)/pH8.5 (external) are the same in a. c.

474 Quench of fluorescence under symmetrical $\mathrm{KCl}$, symmetrical $\mathrm{NaCl}$, and symmetrical K-

475 Gluconate. d. Schematic view of a TsFpn containing proteoliposome for monitoring $\mathrm{H}^{+}$influx. e.

476 Quench of pyranine fluorescence after addition of EDTA to the external solution. f. Schematic

477 view of a TsFpn containing proteoliposome with calcein dye enclosed. $\mathrm{KCl}$ is $100 \mathrm{mM}$ inside of

478 the liposomes and $1 \mathrm{mM}$ outside. Valinomycin is added to clamp the membrane potential at -

$479120 \mathrm{mV}$. g. Quench of calcein fluorescence over time measured with and without addition of

480 valinomycin under the conditions shown in f. h. $\mathrm{Co}^{2+}$ transport of S1 and S2 mutants under 
bioRxiv preprint doi: https://doi.org/10.1101/2020.03.04.975748; this version posted March 5, 2020. The copyright holder for this preprint (which was not certified by peer review) is the author/funder, who has granted bioRxiv a license to display the preprint in perpetuity. It is made available under aCC-BY-NC-ND 4.0 International license.

481 symmetrical and unsymmetrical $\mathrm{pH}$. i. Quench of calcein fluorescence over time measured with

482 and without addition of valinomycin under the conditions shown in $\mathbf{f}$. $\mathbf{j}$. Quench of pyranine

483 fluorescence for S1 and S2 mutants.

484 

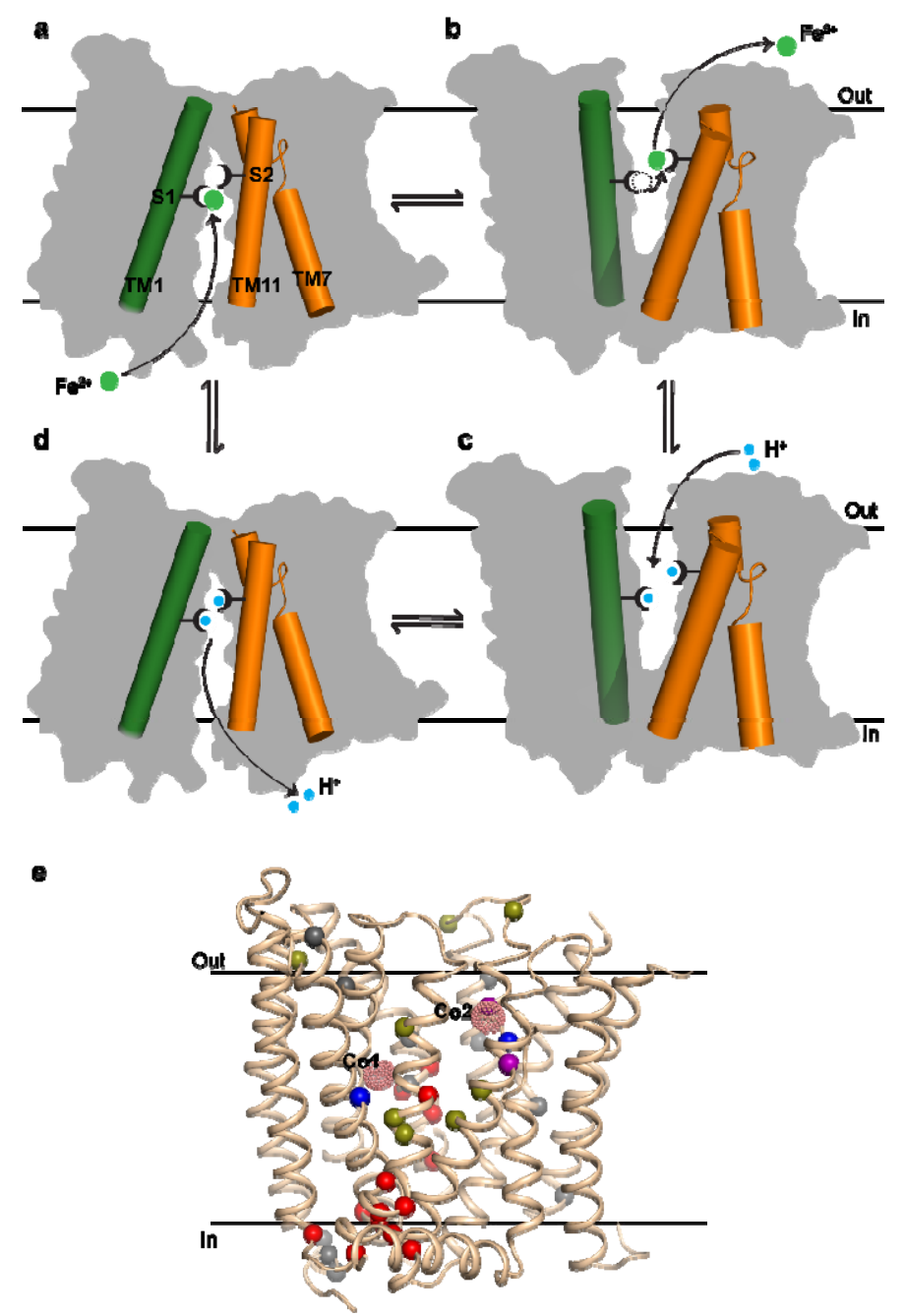

Figure 5. Proposed mechanism of ion transport and disease-related mutations in Fpn. a-d.

487 Proposed conformational changes of Fpn during the transport cycle. A cytosolic $\mathrm{Fe}^{2+}$ ion (green sphere) binds to either the S1 or S2 sites (drawn as forks) of a Fpn at the inward-facing conformation (a); Fpn then switches to the outward-facing conformation to allow the bound $\mathrm{Fe}^{2+}$ to escape to the extracellular side (b). Two protons bind to the S1 and S2 sites in an outward-

491 facing conformation of Fpn (c) and enter the cytosol when Fpn returns to the inward-facing 492 conformation (d). e. Cartoon representation of TsFpn. The bound $\mathrm{Co}^{2+}$ ions are shown as pink 493 dots. $\mathrm{C} \alpha$ of disease-related missense mutations are shown as spheres. Mutations close to the ion 494 binding sites are colored blue, those at the interface between the $\mathrm{N}$ and $\mathrm{C}$ domains are colored 
bioRxiv preprint doi: https://doi.org/10.1101/2020 03.04.975748; this version posted March 5, 2020. The copyright holder for this preprint (which was not certified by peer review) is the author/funder, who has granted bioRxiv a license to display the preprint in perpetuity. It is made available under aCC-BY-NC-ND 4.0 International license.

495 red, and ones that are reported to affect hepcidin binding and endocytosis are colored olive.

496 Cys326 and His508 are shown in purple. All other mutations are colored in grey. 


\section{Extended Data}

a

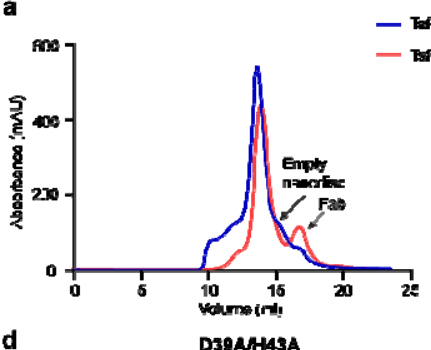

d

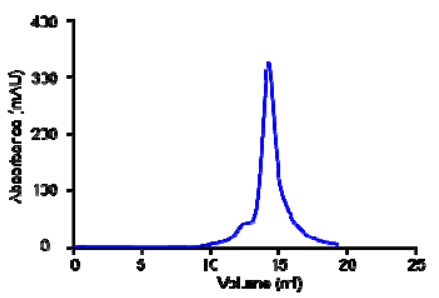

b

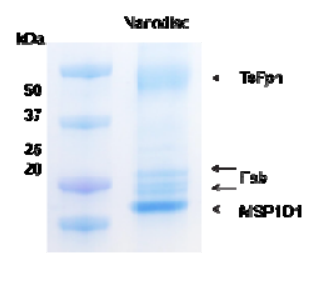

e



c

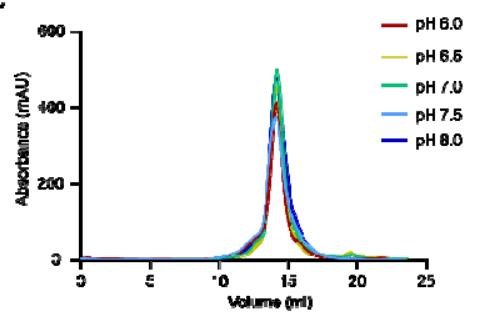

$\mathbf{f}$

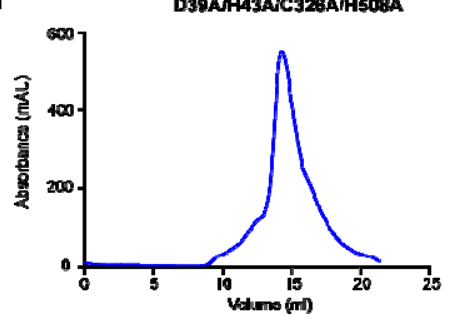

Extended Data Figure 1. Wild type and mutant TsFpn proteins. a. Size-exclusion

501 chromatography of TsFpn in complex with Fab of 11F9 before and after reconstitution into

502 nanodiscs. b. SDS-PAGE of the reconstitution. c. Size-exclusion chromatography of TsFpn in

$503 \mathrm{pH}$ ranging from 6.0 to 8.5. d-f. Size-exclusion chromatography of TsFpn with S1 (D39A/H43A),

504 S2 (C326A/H508A) and S1+S2 (D39A/H43A/C326A/H508A) mutations. 


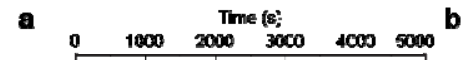
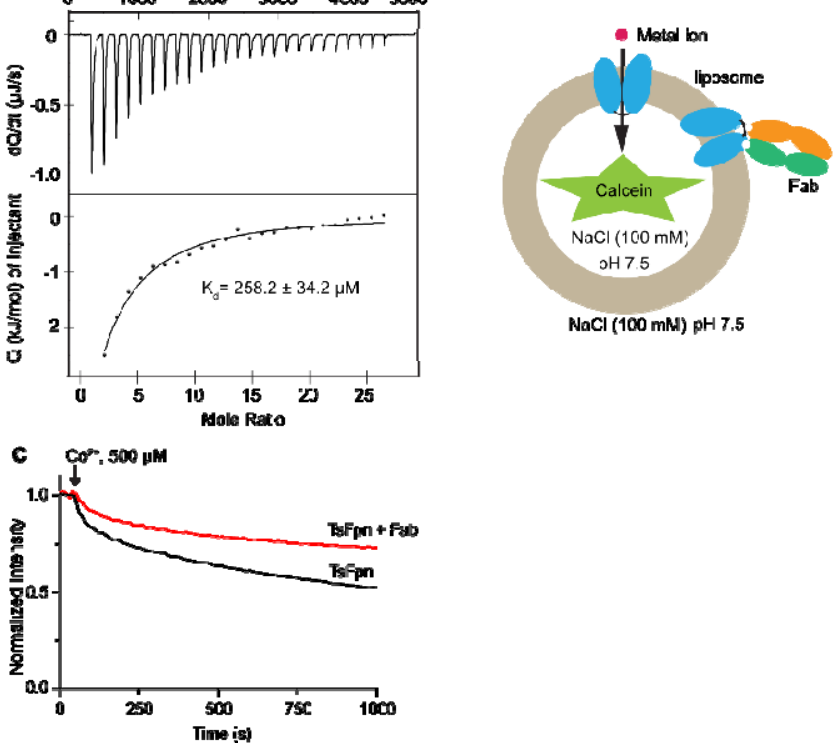

507 Extended Data Figure 2. Effect of Fab on $\mathrm{Co}^{2+}$ binding and transport. a. ITC measurement

508 of $\mathrm{Co}^{2+}$ binding to TsFpn in the presence of $11 \mathrm{~F} 9 \mathrm{Fab}$. b. Schematic illustration of a

509 proteoliposome with TsFpn in both orientations. The liposomes are loaded with calcein and the 1

$510 \mu \mathrm{M}$ of 11F9 Fab was added to the external side of the liposomes. c. Quench of calcein

511 fluorescence in the absence (black trace) and presence of $1 \mu \mathrm{M} 11 \mathrm{~F} 9 \mathrm{Fab}$ (red trace). 


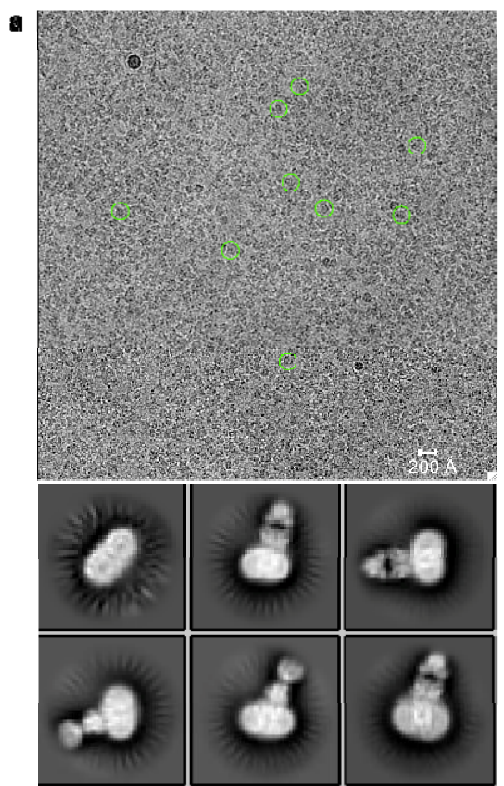

d

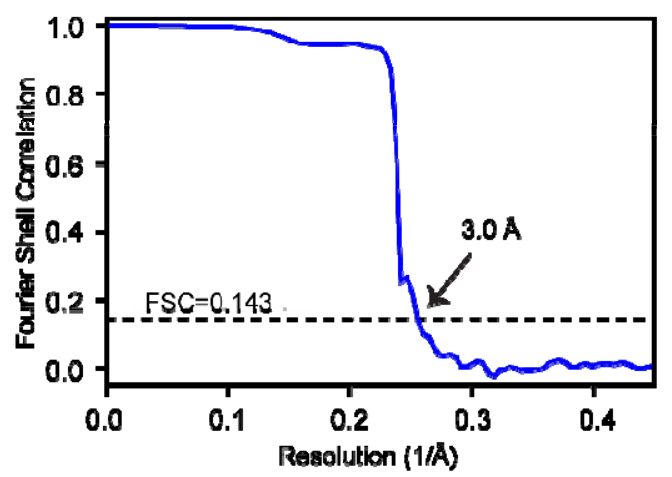

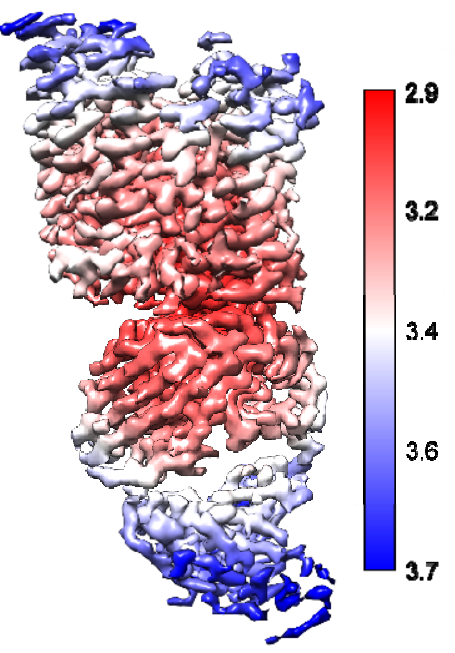

-

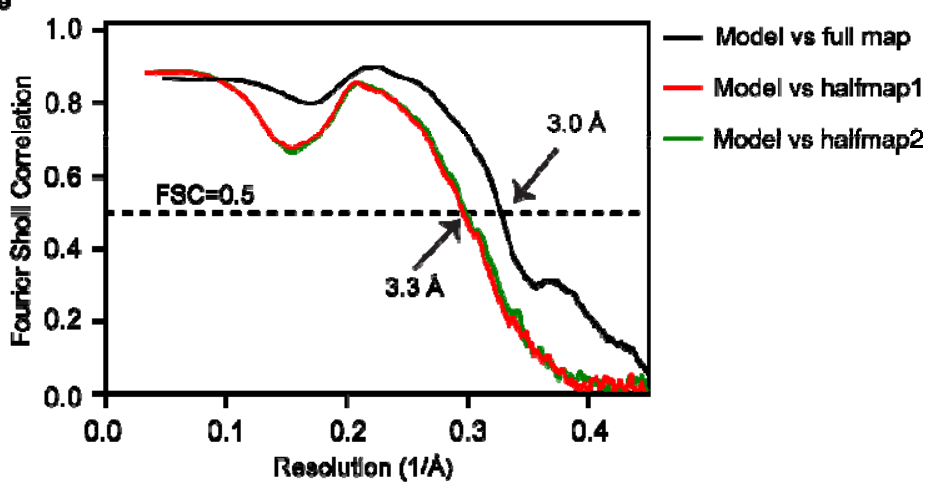

Extended Data Figure 3. Cryo-EM analysis of the TsFpn-Fab complex reconstituted in

nanodisc. a. Representative electron micrograph and 2D class averages of cryo-EM particle

517 images. b. Local resolution map for the 3D reconstruction of the TsFpn-Fab complex. c. Euler angle distribution of the TsFpn-Fab complex in the final 3D reconstruction. d. The gold-standard Fourier shell correlation curve for the final map. e. FSC curve of the refined model of the TsFpnFab complex versus the full map (black) and individual halfmaps (red and green). 
bioRxiv preprint doi: https://doi. org/10.1101/2020 03.04 975748. this version posted March 5,2020 . The copyright holder for this preprint (which was not certified by peer review) is the author/funder, who has granted bioRxiv a license to display the preprint in perpetuity. It is made available under aCC-BY-NC-ND 4.0 International license.

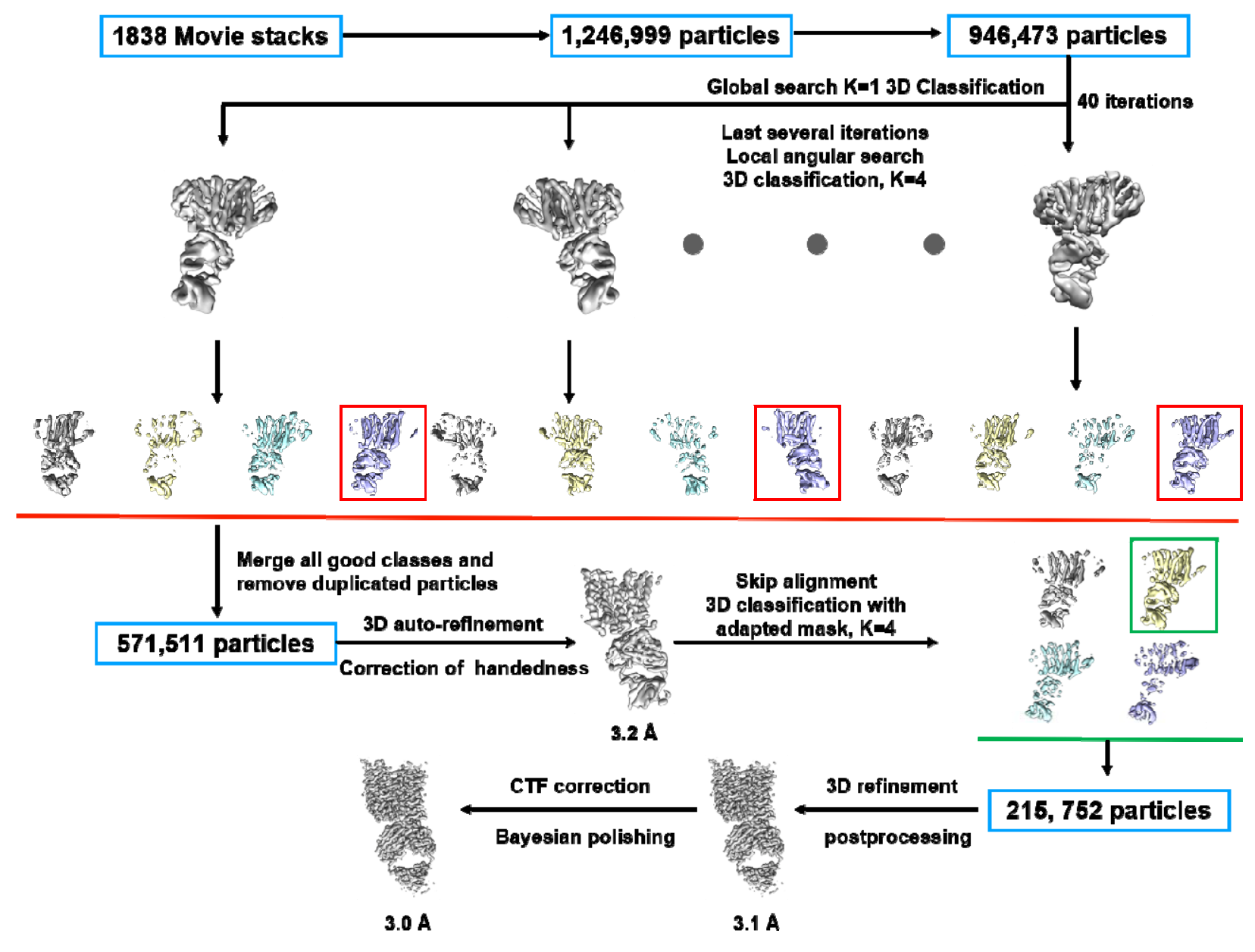

524 Extended Data Figure 4. Flow chart of Cryo-EM data processing. 



TM1 (25-52)

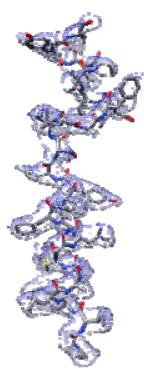

TM7 (306-332)

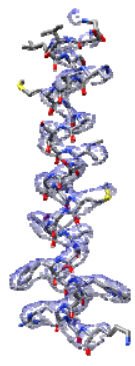

TM8 (339-366)
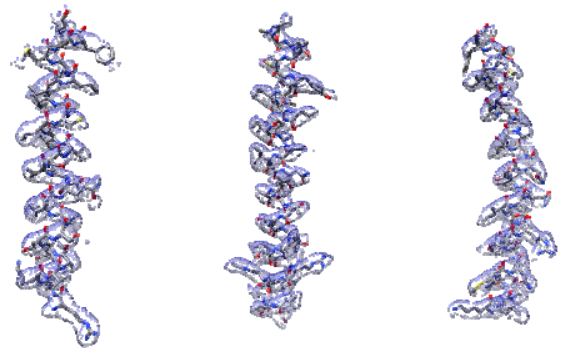

TM3 (88-115)

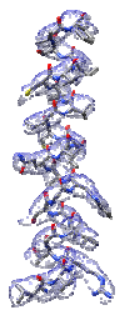

TM9 (369-392)
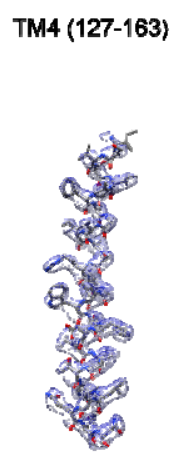

TM10 (452-48L)
TM5 (168-199)

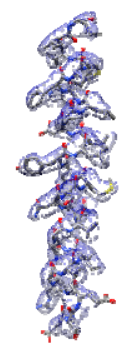

TM11 (487-514)

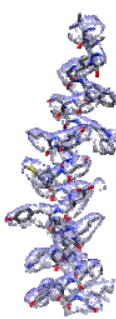

TM6 (202-230)

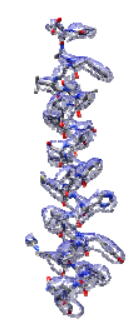

TM12 (518-545) 



B, bacterievorus

\section{T. syrichta \\ H. sapiens \\ D. nario \\ B. bacteriovorus}
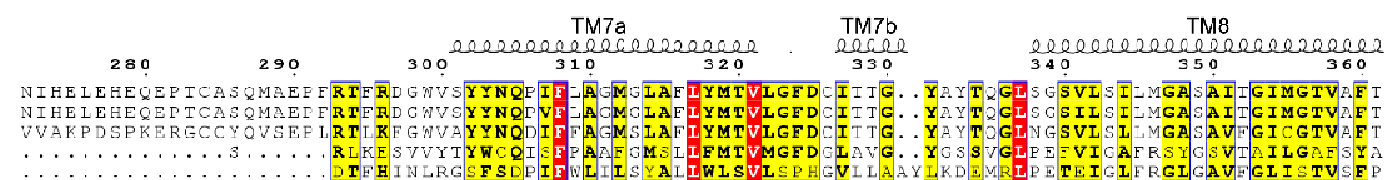

330

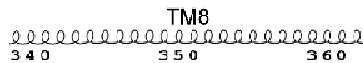

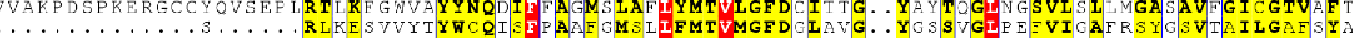

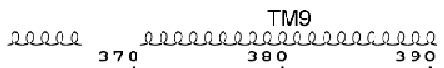
400 410 420 430 440

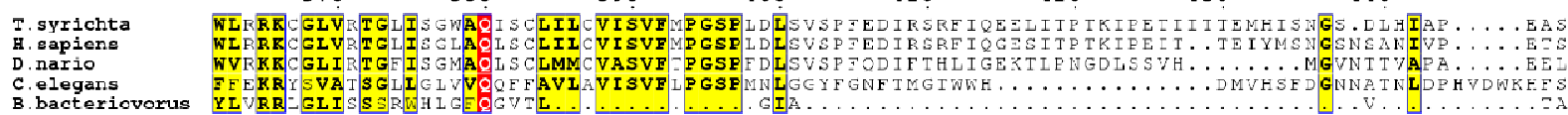

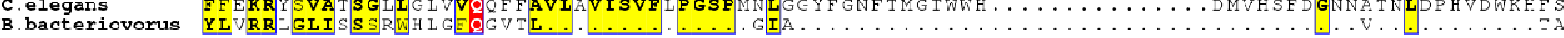


532 Extended Data Figure 6. Fpn sequence alignment. a. Fpn from Philippine tarsier (Uniprot 

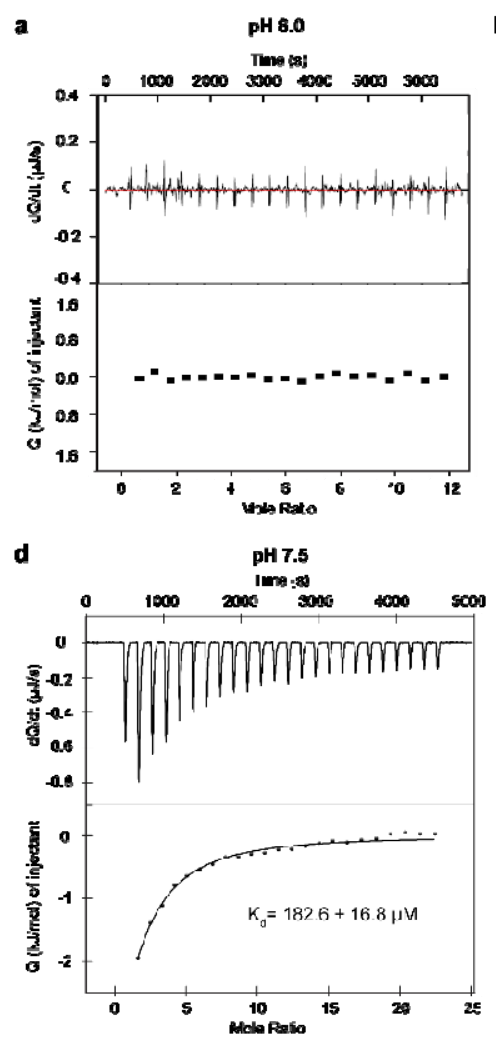

$\mathbf{g}$
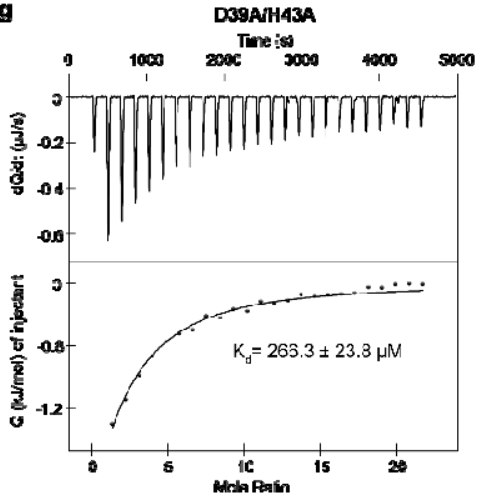

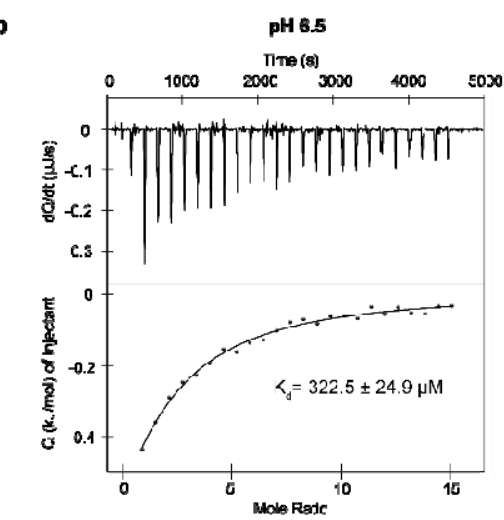

•
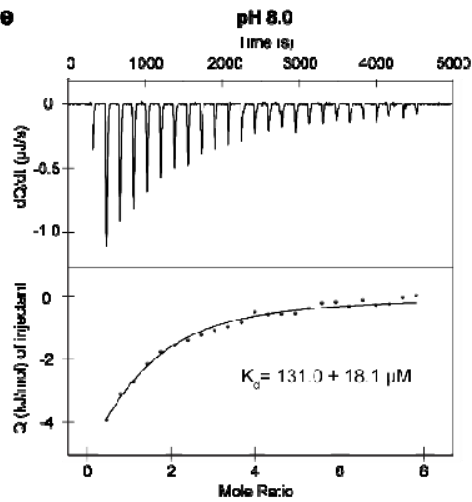

h

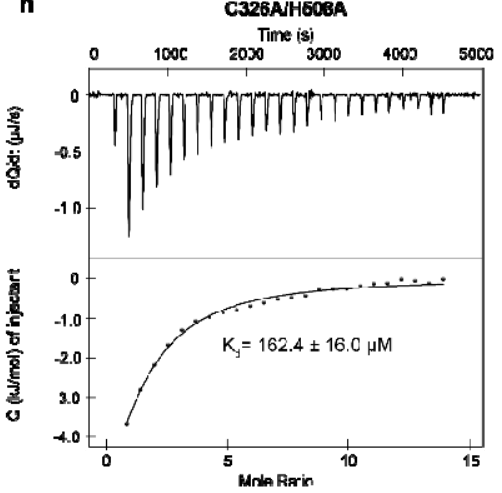

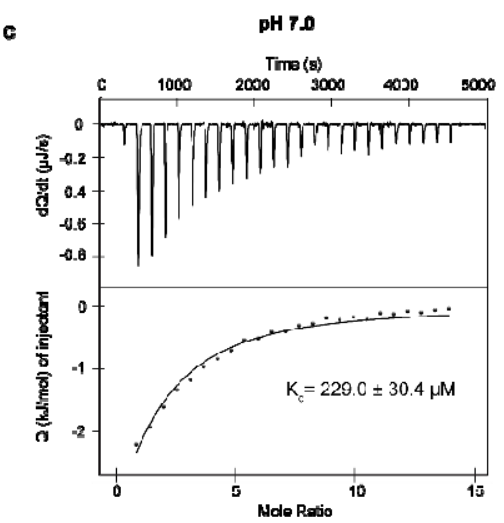

f

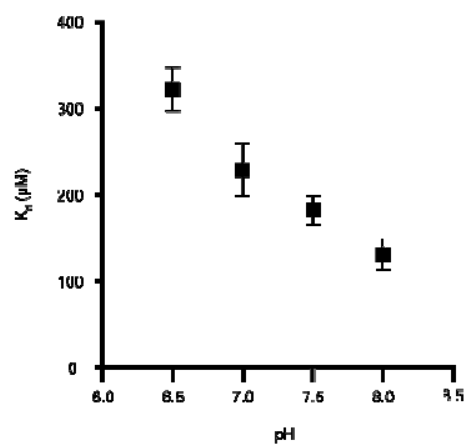

I

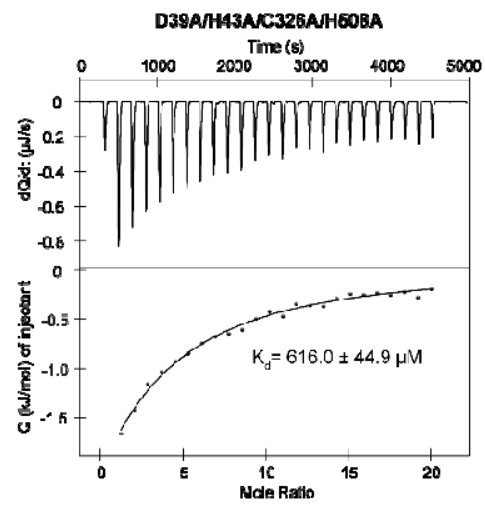

Extended Data Figure 7. ITC measurements of $\mathrm{Co}^{2+}$ binding in different pH. ITC measurements of $\mathrm{Co}^{2+}$ binding to TsFpn in pH $6.0(\mathbf{a}) ; \mathrm{pH} 6.5(\mathbf{b}) ; \mathrm{pH} 7.0(\mathbf{c}) ; \mathrm{pH} 7.5(\mathbf{d}) ; \mathrm{pH} 8.0$ (e). f. $\mathrm{K}_{\mathrm{d}}$ of $\mathrm{Co}^{2+}$ versus different $\mathrm{pH}$. Error bars are s.e.m.. In a-e, the top panel is the rate of heat release versus time and the bottom panel is heat from each injection versus the molar ratios

543 between $\mathrm{Co}^{2+}$ and TsFpn. Data points in b-e were fit with a single-binding site equation. ITC

544 binding experiment at each $\mathrm{pH}$ was repeated three times the composite $\mathrm{K}_{\mathrm{d}}$ values were plotted in

545 f. g-i. ITC measurements of $\mathrm{Co}^{2+}$ binding to $\mathrm{S} 1(\mathbf{g}), \mathrm{S} 2$ (h) and $\mathrm{S} 1+\mathrm{S} 2$ (i) mutants. 
a

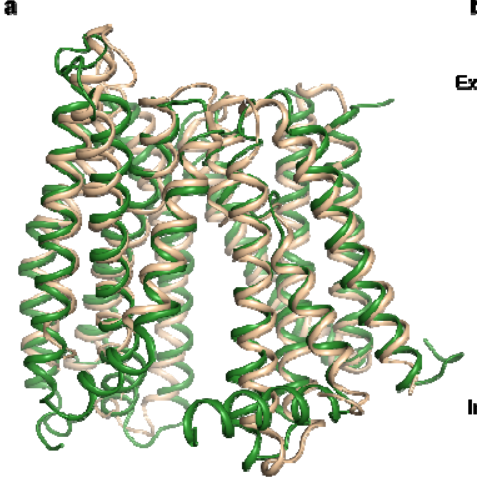

b

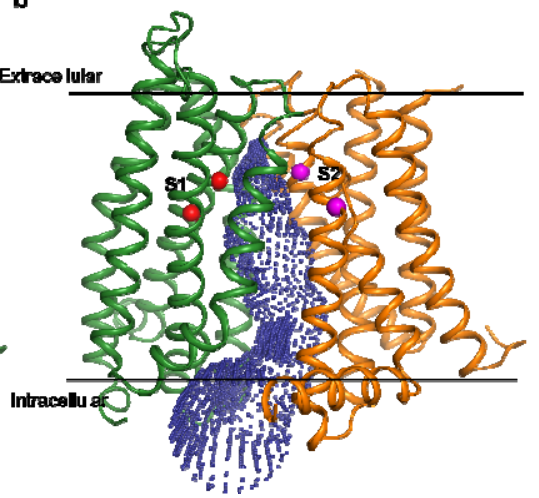

c
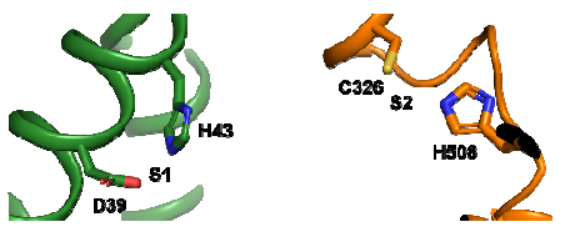

548 Extended Data Figure 8. Inward-facing model of TsFpn. a. The inward-facing model of

549 TsFpn (cartoon in green) is superposed onto BbFpn (cartoon in wheat) in two views. b. cartoon

550 representation of the inward-facing model of TsFpn with the $\mathrm{N}$ - and C-domains shown in green

551 and orange, respectively. The solvent accessible regions in the cavity, calculated by $\mathrm{HOLE}^{52}$, is

552 shown as blue dots. C-alphas of S1 and S2 are shown as spheres and colored red and magenta,

553 respectively. c. Close view of S1 and S2 with side chains shown as sticks. 
a
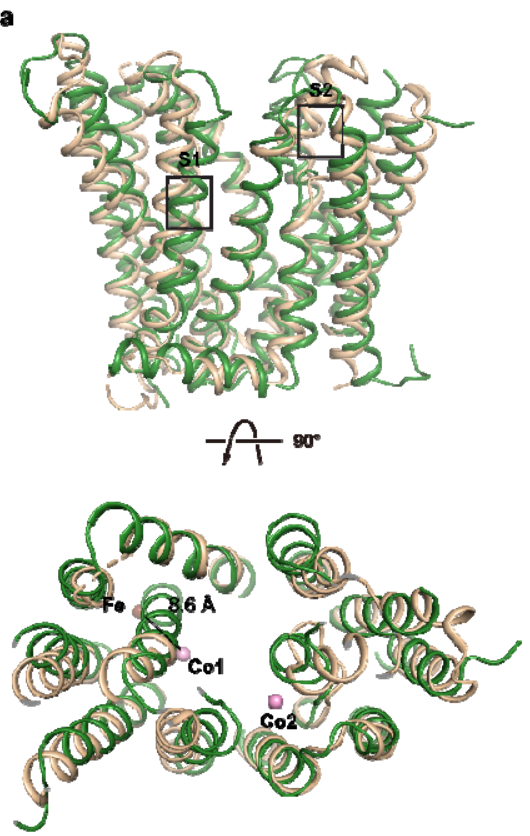

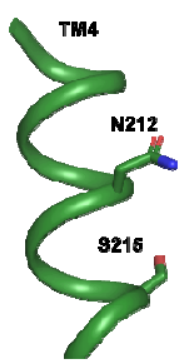

d

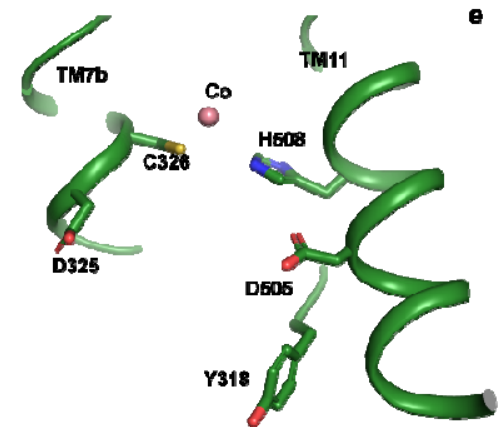

c
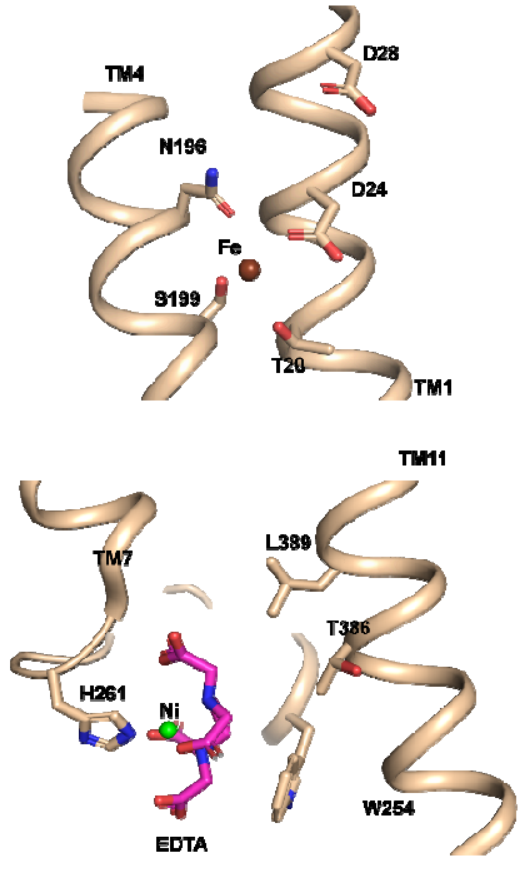

Extended Data Figure 9. Comparison of ion binding sites in TsFpn and BbFpn. a. TsFpn

557 (cartoon in green) is superposed onto BbFpn (cartoon in wheat) in two views. b and d. Ion

558 binding sites in TsFpn. c and e. Ion binding sites in BbFpn. 
bioRxiv preprint doi: https://doi.org/10.1101/2020.03.04.975748; this version posted March 5, 2020. The copyright holder for this preprint (which was not certified by peer review) is the author/funder, who has granted bioRxiv a license to display the preprint in perpetuity. It is made available under aCC-BY-NC-ND 4.0 International license.

560 Extended Data Table 1 | Summary of Cryo-EM data collection, processing and structure

561 refinement

\begin{tabular}{ll}
\hline Protein & TsFpn-Fab \\
\hline Cryo-EM Data Collection & 300 \\
Voltage $(\mathrm{kV})$ & 105,000 \\
Magnification $(\mathrm{x})$ & 1.114 \\
Pixel Size $(\AA)$ & 1.56 \\
Electron exposure $\left(\mathrm{e}-/ \AA^{2} /\right.$ frame $)$ & {$[-2.0,-1.2]$} \\
Defocus range $(\mu \mathrm{m})$ & 1838 \\
Number of image stacks & 32 \\
Number of frames per stack & \\
\hline Cryo-EM Data Processing & 946,473 \\
Initial number of particles & 215,752 \\
Final number of particles & $\mathrm{C1}$ \\
Symmetry imposed & -100 \\
Map sharpening B factor $\left(\AA^{2}\right)$ & 3.0 \\
Map resolution $(\AA)$ & $2.9-3.7$ \\
Map resolution range $(\AA)$ & 0.143 \\
FSC threshold & \\
\hline Model Refinement & 847 \\
Number of amino acids & 5392 \\
Total non-hydrogen atoms & 49.83 \\
Average B factor $\left(\AA^{2}\right)$ & 0.005 \\
Bond length r.m.s.d. $(\AA)$ & 0.814 \\
Bond angle r.m.s.d. $\left({ }^{\circ}\right)$ & \\
Ranmachandran Plot & 92.65 \\
Favored $(\%)$ & 7.35 \\
Allowed $(\%)$ & 0.00 \\
Outliers $(\%)$ & 0.28 \\
Rotamer outliers $(\%)$ & 2.56 \\
EMRinger Score & \\
\hline &
\end{tabular}


565

5661 Drakesmith, H., Nemeth, E. \& Ganz, T. Ironing out Ferroportin. Cell Metab 22, 777-787,

567

568

569

570

571

572

5734

574

575

576

$577 \quad 6$

578

$579 \quad 7$

580

581

582

583

584

585

586

587

588

589

590

591

592

593

$594 \quad 14$

595

$596 \quad 15$

597

\section{Reference}

$$
\text { doi:10.1016/j.cmet.2015.09.006 (2015). }
$$

2 Donovan, A. et al. The iron exporter ferroportin/SIc40a1 is essential for iron homeostasis. Cell Metabolism 1, 191-200, doi:10.1016/j.cmet.2005.01.003 (2005).

3 Aschemeyer, S. et al. Structure-function analysis of ferroportin defines the binding site and an alternative mechanism of action of hepcidin. Blood 131, 899-910, doi:10.1182/blood-2017-05786590 (2018).

4 Nemeth, E. et al. Hepcidin Regulates Cellular Iron Efflux by Binding to Ferroportin and Inducing Its Internalization. Science 306, 2090, doi:10.1126/science.1104742 (2004).

5 Pietrangelo, A. Ferroportin disease: pathogenesis, diagnosis and treatment. Haematologica 102, 1972-1984, doi:10.3324/haematol.2017.170720 (2017).

6 Ganz, T. Systemic Iron Homeostasis. Physiological Reviews 93, 1721-1741, doi:10.1152/physrev.00008.2013 (2013).

7 Sebastiani, G., Wilkinson, N. \& Pantopoulos, K. Pharmacological Targeting of the Hepcidin/Ferroportin Axis. Front Pharmacol 7, 160-160, doi:10.3389/fphar.2016.00160 (2016).

8 Crielaard, B. J., Lammers, T. \& Rivella, S. Targeting iron metabolism in drug discovery and delivery. Nat Rev Drug Discov 16, 400-423, doi:10.1038/nrd.2016.248 (2017).

9 Ruchala, P. \& Nemeth, E. The pathophysiology and pharmacology of hepcidin. Trends Pharmacol Sci 35, 155-161, doi:10.1016/j.tips.2014.01.004 (2014).

10 McKie, A. T. et al. A Novel Duodenal Iron-Regulated Transporter, IREG1, Implicated in the Basolateral Transfer of Iron to the Circulation. Molecular Cell 5, 299-309, doi:10.1016/S10972765(00)80425-6 (2000).

11 Donovan, A. et al. Positional cloning of zebrafish ferroportin1 identifies a conserved vertebrate iron exporter. Nature 403, 776-781, doi:10.1038/35001596 (2000).

12 Abboud, S., ๆ, Haile, D. J. \& ๆ. A Novel Mammalian Iron-regulated Protein Involved in Intracellular Iron Metabolism. Journal of Biological Chemistry 275, 19906-19912 (2000).

13 Deng, D. et al. Crystal structure of the human glucose transporter GLUT1. Nature 510, 121-125, doi:10.1038/nature13306 (2014).

14 Deng, D. et al. Molecular basis of ligand recognition and transport by glucose transporters. Nature 526, 391-396, doi:10.1038/nature14655 (2015).

15 Nomura, N. et al. Structure and mechanism of the mammalian fructose transporter GLUT5. Nature 526, 397-401, doi:10.1038/nature14909 (2015). 
$598 \quad 16$

599

600

601

602

603

$604 \quad 18$

605

$606 \quad 19$

607

608

609

610

611

$612 \quad 21$

613

614

615

616

617

$618 \quad 23$

$619 \quad 24$

620

$621 \quad 25$

622

623

$624 \quad 26$

625

626

$627 \quad 27$

628

$629 \quad 28$

630

631

Doki, S. et al. Structural basis for dynamic mechanism of proton-coupled symport by the peptide transporter POT. Proceedings of the National Academy of Sciences 110, 11343, doi:10.1073/pnas.1301079110 (2013).

17 Newstead, S. et al. Crystal structure of a prokaryotic homologue of the mammalian oligopeptide-proton symporters, PepT1 and PepT2. The EMBO Journal 30, 417-426, doi:10.1038/emboj.2010.309 (2011).

8 Solcan, N. et al. Alternating access mechanism in the POT family of oligopeptide transporters. The EMBO Journal 31, 3411-3421, doi:10.1038/emboj.2012.157 (2012).

9 Wright, N. J. \& Lee, S.-Y. Structures of human ENT1 in complex with adenosine reuptake inhibitors. Nature structural \& molecular biology 26, 599-606, doi:10.1038/s41594-019-0245-7 (2019).

20 Madej, M. G., Sun, L., Yan, N. \& Kaback, H. R. Functional architecture of MFS D-glucose transporters. Proceedings of the National Academy of Sciences of the United States of America 111, E719-E727, doi:10.1073/pnas.1400336111 (2014).

21 Taniguchi, R. et al. Outward- and inward-facing structures of a putative bacterial transitionmetal transporter with homology to ferroportin. Nature communications 6, 8545-8545, doi:10.1038/ncomms9545 (2015).

22 Deshpande, C. N. et al. Calcium is an essential cofactor for metal efflux by the ferroportin transporter family. Nature communications 9, 3075-3075, doi:10.1038/s41467-018-05446-4 (2018).

3 Gupta, R., Jung, E. \& Brunak, S. Prediction of N-glycosylation sites in human proteins. (2004).

Wu, S. et al. Fabs enable single particle cryoEM studies of small proteins. Structure (London, England : 1993) 20, 582-592, doi:10.1016/j.str.2012.02.017 (2012).

25 Quistgaard, E. M., Löw, C., Guettou, F. \& Nordlund, P. Understanding transport by the major facilitator superfamily (MFS): structures pave the way. Nature Reviews Molecular Cell Biology 17, 123-132, doi:10.1038/nrm.2015.25 (2016).

26 Rice, A. E., Mendez, M. J., Hokanson, C. A., Rees, D. C. \& Björkman, P. J. Investigation of the biophysical and cell biological properties of ferroportin, a multipass integral membrane protein iron exporter. Journal of molecular biology 386, 717-732, doi:10.1016/j.jmb.2008.12.063 (2009).

27 von Heijne, G. \& Gavel, Y. Topogenic signals in integral membrane proteins. European Journal of Biochemistry 174, 671-678, doi:10.1111/j.1432-1033.1988.tb14150.x (1988).

$28 \mathrm{Ka}$, C. et al. The SLC40A1 R178Q mutation is a recurrent cause of hemochromatosis and is associated with a novel pathogenic mechanism. Haematologica 103, 1796-1805, doi:10.3324/haematol.2018.189845 (2018). 
63229 Vlasveld, L. T. et al. Twenty Years of Ferroportin Disease: A Review or An Update of Published

633

634

63530

636

637

$638 \quad 31$

639

640

641

642

643

644

645

646

$647 \quad 35$

648

$649 \quad 36$

650

651

652

653

654

655

39

656

$657 \quad 40$

658

659

660

661

662

42

663

$664 \quad 43$

665

Clinical, Biochemical, Molecular, and Functional Features. Pharmaceuticals (Basel) 12, 132, doi:10.3390/ph12030132 (2019).

30 Ehrnstorfer, I. A., Geertsma, E. R., Pardon, E., Steyaert, J. \& Dutzler, R. Crystal structure of a SLC11 (NRAMP) transporter reveals the basis for transition-metal ion transport. Nature Structural \& Molecular Biology 21, 990-996, doi:10.1038/nsmb.2904 (2014).

1 Bozzi, A. T. et al. Crystal Structure and Conformational Change Mechanism of a Bacterial NrampFamily Divalent Metal Transporter. Structure (London, England : 1993) 24, 2102-2114, doi:10.1016/j.str.2016.09.017 (2016).

32 Bai, Y. et al. X-ray structure of a mammalian stearoyl-CoA desaturase. Nature 524, 252-256, doi:10.1038/nature14549 (2015).

33 Martens, C. et al. Lipids modulate the conformational dynamics of a secondary multidrug transporter. Nature structural \& molecular biology 23, 744-751, doi:10.1038/nsmb.3262 (2016).

34 Autzen, H. E. et al. Structure of the human TRPM4 ion channel in a lipid nanodisc. Science (New York, N.Y.) 359, 228-232, doi:10.1126/science.aar4510 (2018).

35 Mastronarde, D. N. Automated electron microscope tomography using robust prediction of specimen movements. J Struct Biol 152, 36-51, doi:10.1016/j.jsb.2005.07.007 (2005).

36 Zheng, S. Q. et al. MotionCor2: anisotropic correction of beam-induced motion for improved cryo-electron microscopy. Nat Methods 14, 331-332, doi:10.1038/nmeth.4193 (2017).

37 Grant, T. \& Grigorieff, N. Measuring the optimal exposure for single particle cryo-EM using a 2.6 A reconstruction of rotavirus VP6. Elife 4, e06980, doi:10.7554/eLife.06980 (2015).

38 Zhang, K. Gctf: Real-time CTF determination and correction. J Struct Biol 193, 1-12, doi:10.1016/j.jsb.2015.11.003 (2016).

39 Scheres, S. H. Semi-automated selection of cryo-EM particles in RELION-1.3. J Struct Biol 189, 114-122, doi:10.1016/j.jsb.2014.11.010 (2015).

40 Scheres, S. H. RELION: implementation of a Bayesian approach to cryo-EM structure determination. J Struct Biol 180, 519-530, doi:10.1016/j.jsb.2012.09.006 (2012).

41 Kimanius, D., Forsberg, B. O., Scheres, S. H. \& Lindahl, E. Accelerated cryo-EM structure determination with parallelisation using GPUs in RELION-2. Elife 5, doi:10.7554/eLife.18722 (2016).

42 Zivanov, J. et al. New tools for automated high-resolution cryo-EM structure determination in RELION-3. elife 7, e42166, doi:10.7554/eLife.42166 (2018).

43 Rosenthal, P. B. \& Henderson, R. Optimal determination of particle orientation, absolute hand, and contrast loss in single-particle electron cryomicroscopy. J Mol Biol 333, 721-745 (2003). 
666

667

668

669

670

671

$672 \quad 46$

673

674

675

676

677

678

679

680

681

682

$683 \quad 50$

684

685

686

687

688

689

690
44 Chen, S. et al. High-resolution noise substitution to measure overfitting and validate resolution in 3D structure determination by single particle electron cryomicroscopy. Ultramicroscopy 135, 24-35, doi:10.1016/j.ultramic.2013.06.004 (2013).

45 Emsley, P., Lohkamp, B., Scott, W. G. \& Cowtan, K. Features and development of Coot. Acta crystallographica. Section D, Biological crystallography 66, 486-501, doi:10.1107/S0907444910007493 (2010).

46 Adams, P. D. et al. PHENIX: a comprehensive Python-based system for macromolecular structure solution. Acta crystallographica. Section D, Biological crystallography 66, 213-221, doi:10.1107/S0907444909052925 (2010).

47 Barad, B. A. et al. EMRinger: side chain-directed model and map validation for 3D cryo-electron microscopy. Nature methods 12, 943-946, doi:10.1038/nmeth.3541 (2015).

48 Ren, Z. et al. Structure of an ElIC sugar transporter trapped in an inward-facing conformation. Proceedings of the National Academy of Sciences of the United States of America 115, 59625967, doi:10.1073/pnas.1800647115 (2018).

49 Baker, N. A., Sept, D., Joseph, S., Holst, M. J. \& McCammon, J. A. Electrostatics of nanosystems: Application to microtubules and the ribosome. Proceedings of the National Academy of Sciences 98, 10037 (2001).

50 Sievers, F. et al. Fast, scalable generation of high-quality protein multiple sequence alignments using Clustal Omega. Molecular systems biology 7, 539-539, doi:10.1038/msb.2011.75 (2011).

51 Robert, X. \& Gouet, P. Deciphering key features in protein structures with the new ENDscript server. Nucleic Acids Research 42, W320-W324, doi:10.1093/nar/gku316 (2014).

52 Smart, O. S., Neduvelil, J. G., Wang, X., Wallace, B. A. \& Sansom, M. S. P. HOLE: A program for the analysis of the pore dimensions of ion channel structural models. Journal of Molecular Graphics 14, 354-360, doi:https://doi.org/10.1016/S0263-7855(97)00009-X (1996). 